\title{
CHARACTERIZING HEAVY-TAILED DISTRIBUTIONS INDUCED BY RETRANSMISSIONS
}

\author{
PREDRAG R. JELENKOVIĆ, ${ }^{*}$ Columbia University \\ JIAN TAN, ${ }^{* *}$ IBM T. J. Watson Research Center
}

\begin{abstract}
Consider a generic data unit of random size $L$ that needs to be transmitted over a channel of unit capacity. The channel availability dynamic is modeled as an independent and identically distributed sequence $\left\{A, A_{i}\right\}_{i \geq 1}$ that is independent of $L$. During each period of time that the channel becomes available, say $A_{i}$, we attempt to transmit the data unit. If $L<A_{i}$, the transmission is considered successful; otherwise, we wait for the next available period $A_{i+1}$ and attempt to retransmit the data from the beginning. We investigate the asymptotic properties of the number of retransmissions $N$ and the total transmission time $T$ until the data is successfully transmitted. In the context of studying the completion times in systems with failures where jobs restart from the beginning, it was first recognized by Fiorini, Sheahan and Lipsky (2005) and Sheahan, Lipsky, Fiorini and Asmussen (2006) that this model results in power-law and, in general, heavy-tailed delays. The main objective of this paper is to uncover the detailed structure of this class of heavy-tailed distributions induced by retransmissions. More precisely, we study how the functional relationship $\mathbb{P}[L>x]^{-1} \approx \Phi\left(\mathbb{P}[A>x]^{-1}\right)$ impacts the distributions of $N$ and $T$; the approximation ' $\approx$ ' will be appropriately defined in the paper based on the context. Depending on the growth rate of $\Phi(\cdot)$, we discover several criticality points that separate classes of different functional behaviors of the distribution of $N$. For example, we show that if $\log (\Phi(n))$ is slowly varying then $\log (1 / \mathbb{P}[N>n])$ is essentially slowly varying as well. Interestingly, if $\log (\Phi(n))$ grows slower than $\mathrm{e}^{\sqrt{\log n}}$ then we have the asymptotic equivalence $\log (\mathbb{P}[N>n]) \approx-\log (\Phi(n))$. However, if $\log (\Phi(n))$ grows faster than $\mathrm{e}^{\sqrt{\log n}}$, this asymptotic equivalence does not hold and admits a different functional form. Similarly, different types of distributional behavior are shown for moderately heavy tails (Weibull distributions) where $\log (\mathbb{P}[N>n]) \approx$ $-(\log \Phi(n))^{1 /(\beta+1)}$, assuming that $\log \Phi(n) \approx n^{\beta}$, as well as the nearly exponential ones of the form $\log (\mathbb{P}[N>n]) \approx-n /(\log n)^{1 / \gamma}, \gamma>0$, when $\Phi(\cdot)$ grows faster than two exponential scales $\log \log (\Phi(n)) \approx n^{\gamma}$.

Keywords: Retransmission; channel (systems) with failures; restart; origins of heavy tails (subexponentiality); Gaussian distribution; exponential distribution; Weibull distribution; log-normal distribution; power law
\end{abstract}

2010 Mathematics Subject Classification: Primary 60F99

Secondary 60F10; 60G50; 60G99

\section{Introduction}

Retransmissions represent one of the most fundamental approaches in communication networks that guarantee data delivery in the presence of channel failures. These types of

Received 6 May 2011; revision received 8 February 2012.

This work was supported by the NSF grant CCF-0915784.

* Postal address: Department of Electrical Engineering, Columbia University, New York, NY 10027, USA.

** Postal address: IBM T. J. Watson Research, Yorktown Heights, NY 10598, USA. Email address: tanji@us.ibm.com 
mechanisms have been employed in all networking layers, including, for example, the automatic repeat request (ARQ) protocol (see, e.g. Section 2.4 of [2]) in the data link layer [8] where a packet is resent automatically in case of an error; contention-based ALOHA-type protocols in the medium access control (MAC) layer that use random backoff and a retransmission mechanism [7] to recover data from collisions; end-to-end acknowledgement for multi-hop transmissions in the transport layer [9]; the HTTP downloading scheme in the application layer, etc. For further discussions of the engineering implications of our results in communication networks, see [7], [8], and the extended version of this paper [9].

As briefly stated in the abstract, we use the following generic channel with failures [8] to model the preceding situations. The channel dynamic is described as an on-off process $\left\{(A, U),\left(A_{i}, U_{i}\right)\right\}_{i \geq 1}$ with alternating periods when the channel is available, $A_{i}$, and unavailable, $U_{i}$, respectively; $\left(A, A_{i}\right)_{i \geq 1}$ and $\left(U, U_{i}\right)_{i \geq 1}$ are two independent sequences of independent and identically distributed random variables. During each period of time that the channel becomes available, say $A_{i}$, we attempt to transmit the data unit of random size $L$. If $L<A_{i}$, we say that the transmission is successful; otherwise, we wait for the next period $A_{i+1}$ when the channel is available and attempt to retransmit the data from the beginning. We study the asymptotic properties of the distributions of the total transmission time $T$ and number of retransmissions $N$; for the precise definitions of these variables and the model, see Subsection 1.1.

The preceding model was introduced and studied in [10] and, apart from the already mentioned applications in communications, it represents a generic model for other situations where jobs restart from the beginning after a failure. It was first recognized in [4] that this model results in power-law distributions when the distributions of $L$ and $A$ have a matrix exponential representation, and this result was rigorously proved and further generalized in [13]. Under more general conditions, Jelenković and Tan [8] discovered that the distributions of $N$ and $T$ follow power laws with the same exponent $\alpha$ as long as $\log \mathbb{P}[L>x] \approx \alpha \log \mathbb{P}[A>x]$ for large $x$, which implies that power-law distributions, possibly with infinite mean $(0<\alpha<1)$ and variance $(0<\alpha<2)$, may arise even when transmitting superexponential (e.g. Gaussian) documents/packets. More recent results on the heavy-tailed completion times in a system with failures are developed in [1]. In this paper, we further characterize this class of heavy-tailed distributions that are induced by retransmissions.

From a mathematical perspective, our proofs are based on the method introduced in [8] that uses the following key arguments. First, in exploring the distribution of $N$, we assume that the functional relationship $\Phi(\cdot)$ between the probability distributions $\bar{F}(x)^{-1} \approx \Phi\left(\bar{G}(x)^{-1}\right)$, with $\bar{F}(x):=\mathbb{P}[L>x]$ and $\bar{G}(x):=\mathbb{P}[A>x]$, is eventually monotonically nondecreasing. Then, we use the result that $\bar{F}(L)$ is a uniform random variable on $(0,1)$ given that $\bar{F}(\cdot)$ is absolutely continuous (see [8] and [7]), e.g. for $\bar{F}(x)=(\bar{G}(x))^{\alpha}, \alpha>0$, the key argument on the uniform distribution of $\bar{F}(L)$ from [8] can be illustrated as

$$
\mathbb{P}[N>n]=\mathbb{E}\left[(1-\bar{G}(L))^{n}\right] \approx \mathbb{E}\left[\mathrm{e}^{-n \bar{G}(L)}\right]=\mathbb{E}\left[\mathrm{e}^{-n \bar{F}^{1 / \alpha}(L)}\right] \approx \frac{\Gamma(\alpha+1)}{n^{\alpha}} .
$$

Second, in contrast to [13] and [1], instead of studying the total transmission time $T$ directly, we study a simpler quantity $N$ and then use large deviation techniques to investigate $T$, since $T$ can be represented as a sum of $L$ and $\left\{\left(A_{i}+U_{i}\right)\right\}_{1 \leq i<N}$; see (1) in the next subsection. Hence, our analysis is entirely probabilistic, which differs from the work in [1] that relies on Tauberian theorems.

We extend the results from [1] and [8] under a more unified framework, and study how the functional relationship between the data unit characteristics and channel dynamics in the form 
$\mathbb{P}[L>x]^{-1} \approx \Phi\left(\mathbb{P}[A>x]^{-1}\right)$ impacts the distribution of $N$, where the definition of the approximation ' $\approx$ ' may vary according to the context.

The rest of the paper is organized as follows. After a detailed description of the channel model in Subsection 1.1, we present our main results in Section 2: the asymptotics of the distribution of $N$ in Subsection 2.1 and the asymptotics of the distribution of $T$ in Subsection 2.2. In Subsection 2.1 we study three types of distinct behavior, i.e. the very heavy asymptotics in Subsection 2.1.1, the medium heavy (Weibull) asymptotics in Subsection 2.1.2, and the nearly exponential asymptotics in Subsection 2.1.3. Some of the technical proofs are postponed to Section 3.

\subsection{Description of the channel}

In this section we formally describe our model and provide necessary definitions and notation. Consider transmitting a generic data unit of random size $L$ over a channel with failures. Without loss of generality, we assume that the channel is of unit capacity. The channel dynamic is modeled as an on-off process $\left\{\left(A_{i}, U_{i}\right)\right\}_{i \geq 1}$ with alternating independent periods when the channel is available, $A_{i}$, and unavailable, $U_{i}$, respectively. In each period of time that the channel becomes available, say $A_{i}$, we attempt to transmit the data unit and, if $A_{i}>L$, we say that the transmission is successful; otherwise, we wait for the next period $A_{i+1}$ when the channel is available and attempt to retransmit the data from the beginning. A sketch of the model depicting the system is given in Figure 1.

Assume that $\left\{U, U_{i}\right\}_{i \geq 1}$ and $\left\{A, A_{i}\right\}_{i \geq 1}$ are two mutually independent sequences of independent and identically distributed random variables.

Definition 1. The total number of (re)transmissions for a generic data unit of length $L$ is defined as

$$
N:=\inf \left\{n: A_{n}>L\right\},
$$

and the total transmission time for the data unit is defined as

$$
T:=\sum_{i=1}^{N-1}\left(A_{i}+U_{i}\right)+L .
$$

We respectively denote the complementary cumulative distribution functions for $A$ and $L$ as

$$
\bar{G}(x):=\mathbb{P}[A>x] \quad \text { and } \quad \bar{F}(x):=\mathbb{P}[L>x] .
$$

It was first discovered in Theorem 6 of [13] that this model leads to subexponential delay $T$ under quite general conditions. The following slightly more general proposition was proven in Lemma 1 of [8] using probabilistic arguments (see also Proposition 1.2 of [1]).

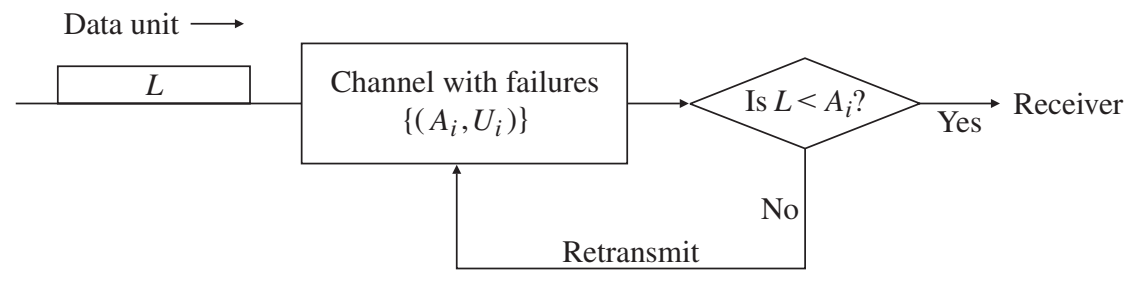

FIGURE 1: Data unit retransmissions over a channel with failures. 
Proposition 1. If $\bar{F}(x)>0$ for all $x \geq 0$, then both $N$ and $T$ are subexponential in the sense that, for any $\varepsilon>0$,

$$
\mathrm{e}^{\varepsilon n} \mathbb{P}[N>n] \rightarrow \infty \text { as } n \rightarrow \infty
$$

and

$$
\mathrm{e}^{\varepsilon t} \mathbb{P}[T>t] \rightarrow \infty \text { as } t \rightarrow \infty .
$$

Clearly, Proposition 1 defines a class of subexponential distributions that are induced by retransmissions; the proof of this proposition can be found in [8]. The main objective of this paper is to uncover the detailed structure of this class of distributions. More precisely, we investigate how the functional dependence of $\bar{F}$ and $\bar{G}$ (stated in the form $\bar{F}(x)^{-1} \approx$ $\left.\Phi\left(\bar{G}(x)^{-1}\right)\right)$ impacts the tail characteristics of the distributions of both $N$ and $T$; the exact meaning of ' $\approx$ ' will be defined according to the context.

In the paper we use the following standard notation. For any two real functions $a(t)$ and $b(t)$, we write $a(t) \sim b(t)$ as $t \rightarrow \infty$ to denote $\lim _{t \rightarrow \infty}[a(t) / b(t)]=1$. Similarly, we say that $a(t) \gtrsim b(t)$ as $t \rightarrow \infty$ if $\underline{\lim }_{t \rightarrow \infty} a(t) / b(t) \geq 1 ; a(t) \lesssim b(t)$ has a complementary definition. In addition, we say that $a(t)=o(b(t))$ as $t \rightarrow \infty$ if $\lim _{t \rightarrow \infty} a(t) / b(t)=0$. We often simply write $a(t)=o(b(t))$ without explicitly stating $t \rightarrow \infty$ in order to simplify the notation. Also, we use the standard definition of a generalized inverse function $f^{\leftarrow}(x):=\inf \{y: f(y)>x\}$ for a function $f(x)$; note that the notation $f(x)^{-1}$ is reserved for $1 / f(x)$.

\section{Main results}

In this section we present our main results. Here, we assume that $\bar{F}(x)$ is an absolutely continuous function with support on $[0, \infty)$, i.e. $\bar{F}(x)>0$ for $x \geq 0$. If $\bar{F}(x)$ is lattice valued, our results may still hold; see Remarks 4 and 7. If $\bar{F}(x)$ has only a finite support, we discuss this situation in Section 3 of the extended version of this paper [9]; see also Example 3 in Section IV of [8] and Section 3 of [1]. According to (1), the total transmission time $T$ naturally depends on the number of transmissions $N$, and, therefore, we first study the distributional properties of the number of transmissions $N$ in Subsection 2.1, and then evaluate the total transmission time $T$ using the large deviation approach in Subsection 2.2. We use the standard notation from the theory of regularly varying functions [3]. A function $l(x): \mathbb{R}^{+} \rightarrow \mathbb{R}^{+}$is slowly varying if, for any $\lambda>0, l(\lambda x) / l(x) \rightarrow 1$ as $x \rightarrow \infty$; and $\Phi(x)=x^{\alpha} l(x)$ is called regularly varying of index $\alpha$. Furthermore, we define the dominantly regularly varying functions.

Definition 2. For an eventually nondecreasing function $\Phi(x): \mathbb{R}^{+} \rightarrow \mathbb{R}^{+}$, we say that $\Phi(x)$ is dominantly regularly varying if

$$
\varlimsup_{x \rightarrow \infty} \frac{\Phi(\mathrm{e} x)}{\Phi(x)}<\infty
$$

where e $\equiv \exp (1)$

Remark 1. Note that $\Phi(x)$ is eventually nondecreasing. Thus, if $\overline{\lim }_{x \rightarrow \infty} \Phi(c x) / \Phi(x)<\infty$ for some constant $c>1$, say $c=\mathrm{e}$, then it holds for all $c>1$. Also, it is known that dominantly regularly varying functions contain the regularly varying functions [3].

Throughout the paper, we assume that $\Phi(x)$ is a positive, locally bounded on $(0, \infty)$, and eventually nondecreasing real function. 


\subsection{Asymptotics of the distribution of the number of retransmissions $N$}

In this subsection we present the asymptotic results for the number of retransmissions $N$ depending on the functional relationship $\Phi(\cdot)$ between $\bar{F}$ and $\bar{G}$. Informally, we study three scenarios, very heavy asymptotics (when $\log (\Phi(n))$ is slowly varying), medium heavy (Weibull) asymptotics (when $\log (\Phi(n))$ is regularly varying), and nearly exponential (when $\log \log (\Phi(n)$ ) is regularly varying), where within and between these subclasses we also identify critical functional points that define different distributional behaviors of $N$.

Informally, the results can be briefly summarized as follows.

- If $\Phi(n)$ is dominantly regularly varying, e.g. regularly varying, $\Phi(n)=n^{\alpha} l(n), \alpha>0$, then $\mathbb{P}[N>n] \approx \Phi(n)^{-1}$, as stated in Proposition 3 and Theorem 1 .

- If $\Phi(n)$ is not dominantly regularly varying, e.g. $\Phi(n)^{-1}$ being lognormal, the preceding tail equivalence $\mathbb{P}[N>n] \approx \Phi(n)^{-1}$ does not hold, as shown in Proposition 4. However, we show in a weaker form that if $\log (\Phi(n))$ is slowly varying then $\log (\mathbb{P}[N>n])$ is essentially slowly varying as well, as proved in Proposition 2 . Interestingly, within this class, we discover two types of distinct functional behavior of $\log \mathbb{P}[N>n]$ depending on the growth rate of $\log (\Phi(n))$.

- If $\log (\Phi(n))$ grows slower than $\mathrm{e}^{\sqrt{\log n}}$, then we have the asymptotic equivalence $\log (\mathbb{P}[N>n]) \approx-\log (\Phi(n))$, as shown in Theorem 2 and Corollary 3, which implies parts $(1: 1),(2: 1)$ and (2:2) of Theorem 2.1 in [1] and extends Theorem 2 in [8].

○ If $\log (\Phi(n))$ grows faster than $\mathrm{e}^{\sqrt{\log n}}$ then the preceding asymptotic equivalence does not hold, and we demonstrate a different functional form of $\mathbb{P}[N>n]$ in Proposition 6.

- If $\log (\Phi(n))$ is regularly varying with index $\beta>0$, then we obtain a Weibull distribution for $N$, i.e. $\log \mathbb{P}[N>n] \approx-(\log \Phi(n))^{1 /(\beta+1)}$, as presented in Theorem 3; we term it moderately heavy (Weibull tail) asymptotics. This result implies part (1:2) of Theorem 2.1 of [1], and provides a more precise logarithmic asymptotic instead of a double logarithmic limit.

- When the decay of $\mathbb{P}[L>x]$ is much faster than $\mathbb{P}[A>x]$, i.e. their distributions are roughly separated by more than two exponential scales, precisely, $\log \log \left(\mathbb{P}[L>x]^{-1}\right) \approx$ $R_{\gamma}\left(\mathbb{P}[A>x]^{-1}\right)$ with $R_{\gamma}(\cdot), \gamma>0$, being regularly varying of index $\gamma$, we obtain nearly exponential distributions for $N$ of the form $\log \left(\mathbb{P}[N>n]^{-1}\right) \approx n / R_{\gamma}^{\leftarrow}(\log n)$ with $R_{\gamma}^{\leftarrow}(\cdot)$ being regularly varying of index $1 / \gamma$, implying that $R_{\gamma}^{\leftarrow}(\log n)$ is slowly varying; see Theorem 4.

After the preceding characterization of the different classes of distributional behaviors for $N$, we study in Subsection 2.2 the total transmission time $T$. As previously stated, for studying $T$, we use the large deviation results since $T$ can be represented as the sum of $L$ and $\left\{\left(A_{i}+U_{i}\right)\right\}_{1 \leq i<N}$. In this context, our primary results show that (i) when $\Phi(\cdot)$ is regularly varying, we derive the exact asymptotics for $T$ in Theorem 5, (ii) when $\log (\Phi(\cdot))$ is slowly varying, we obtain the logarithmic asymptotics for $T$ in Theorem 6 , and (iii) when $\log (\Phi(\cdot))$ is regularly varying with positive index, we derive, in a different scale than in Theorem 6 , the logarithmic asymptotics in Theorem 7 . Note that the preceding three results on $T$ correspond to Theorems 1(i), 2, and 3 on $N$, respectively. Similarly, we can derive the respective statements 
on $\mathbb{P}[T>t]$ for other results on $\mathbb{P}[N>n]$, but we omit this to avoid lengthy expositions and repetitions. Interestingly, we want to point out that the minimum conditions needed for Theorem 7, as shown by Proposition 8 , involve an intriguing balance between the tail decays of $\mathbb{P}[A>x]$ and $\mathbb{P}[L>x]$.

2.1.1. Very heavy asymptotics. In this subsection we study the situation when the distribution of the number of retransmissions $N$ is heavier than Weibull distributions. Specifically, we determine under what conditions $\mathbb{P}[N>n] \approx \Phi(n)^{-1}$ holds, assuming that $\bar{F}(x)^{-1} \approx$ $\Phi\left(\bar{G}(x)^{-1}\right)$, meaning that the complementary cumulative distribution function of $N$ is of the same form (in terms of $\Phi(\cdot)$ ) as the functional relationship $\Phi(\cdot)$ between $\bar{F}$ and $\bar{G}$.

We term this subclass very heavy distributions since if $\log (\Phi(\cdot))$ is slowly varying, then the number of retransmissions $N$ is always heavier than any Weibull distribution; we state this result as a proposition.

Proposition 2. If $\log (\Phi(\cdot))$ is slowly varying and

$$
\lim _{x \rightarrow \infty} \frac{\log \left(\bar{F}(x)^{-1}\right)}{\log \left(\Phi\left(\bar{G}(x)^{-1}\right)\right)}=1,
$$

then, for any $\varepsilon>0$, as $n \rightarrow \infty$,

$$
\lim _{n \rightarrow \infty} \frac{\log \left(\mathbb{P}[N>n]^{-1}\right)}{n^{\varepsilon}}=0 .
$$

The proof of this proposition will be presented in Subsection 3.1. In the remainder of this subsection we study the detailed structure of this class of distributions that have very heavy tails. The Weibull case will be studied in Subsection 2.1.2 on medium heavy asymptotics.

The following two propositions show that $\mathbb{P}[N>n]$ is tail equivalent to $\Phi(n)^{-1}$ basically only when $\Phi(n)$ is dominantly regularly varying.

Proposition 3. If $\Phi(\cdot)$ is dominantly regularly varying and, as $x \rightarrow \infty$,

$$
\bar{F}(x)^{-1} \sim \Phi\left(\bar{G}(x)^{-1}\right),
$$

then, there exists finite $c \geq 1$ such that

$$
c^{-1} \leq \lim _{n \rightarrow \infty} \mathbb{P}[N>n] \Phi(n) \leq \varlimsup_{n \rightarrow \infty} \mathbb{P}[N>n] \Phi(n) \leq c .
$$

Remark 2. Note that, for Proposition 3 as well as the results in the rest of the paper, we could have equivalently assumed that $\bar{F}(x) \sim \Phi(\bar{G}(x))$, where $\Phi(\cdot)$ is eventually nonincreasing and satisfies the appropriate regularity conditions in the neighborhood of 0 , e.g. condition (4) would be restated in the neighborhood of 0 . In this case, the respective statement would be in the form $\mathbb{P}[N>n] \approx \Phi\left(n^{-1}\right)$. We choose the current form since it has additional notational benefits, e.g. $\log \log \Phi(n)$ would need to be replaced by the more cumbersome expression $\log \left(-\log \left(\Phi\left(n^{-1}\right)\right)\right)$ in (say) Proposition 6 below.

Proposition 4. If (4) is satisfied and

$$
\lim _{x \rightarrow \infty} \frac{\Phi(c x)}{\Phi(x)}=\infty
$$

for some $c>1$, then

$$
\lim _{n \rightarrow \infty} \mathbb{P}[N>n] \Phi(n)=\infty
$$


When $\Phi(\cdot)$ is regularly varying, we can compute the exact asymptotics of the distribution of $N$.

Theorem 1. Assume that $\bar{F}(x)^{-1} \sim \Phi\left(\bar{G}(x)^{-1}\right)$, where $\Phi(\cdot)$ is regularly varying with index $\alpha$.

(i) If $\alpha>0$ then, as $n \rightarrow \infty$,

$$
\mathbb{P}[N>n] \sim \frac{\Gamma(\alpha+1)}{\Phi(n)} .
$$

(ii) If $\alpha=0$, i.e. $\Phi(\cdot)$ is slowly varying, then, as $n \rightarrow \infty$,

$$
\mathbb{P}[N>n] \sim \frac{1}{\Phi(n)} .
$$

Remark 3. For $\alpha>0$, Theorem 1 was proved in Theorem 4 of [8] using the method that we further expand in this paper; alternatively, a similar result for $T$ was proved using the Tauberian method in Theorem 2.2 of [1]. We will prove the corresponding result for $T$ in Theorem 5 in Subsection 2.2.

Remark 4. (Lattice variables.) Note that if $\bar{F}(x)$ and $\bar{G}(x)$ are lattice valued, then the distribution of $N$ may still be tail equivalent to $\Phi(n)^{-1}$, as in Proposition 4, but the constant in front of $\Phi(n)^{-1}$ may be different from $\Gamma(\alpha+1)$, e.g. if $\mathbb{P}[L>n] \sim \mathrm{e}^{-p n}, p>0$, and $\mathbb{P}[A>n] \sim \mathrm{e}^{-q n}, q>0$, then this constant is between $\mathrm{e}^{-p} \Gamma(1+p / q)$ and $\mathrm{e}^{p} \Gamma(1+p / q)$.

Before turning to the proof of Theorem 1, we state two straightforward consequences of the preceding theorems; see also Theorem 1 and Corollary 1 of [8]. The following corollary allows $\bar{F}$ and $\bar{G}$ to have exponential-type distributions; the corresponding result for $T$ was first derived in Theorem 7 of [13].

Corollary 1. Assume that $\bar{G}(x) \sim \mathrm{e}^{-\beta x}$ and $\bar{F}(x) \sim a x^{b} \mathrm{e}^{-\delta x}$, where $b \in \mathbb{R}$ and $a, \beta>0$. Then

$$
\mathbb{P}[N>n] \sim a \Gamma\left(\frac{\delta}{\beta}+1\right) \beta^{-b} \frac{(\log t)^{b}}{t^{\delta / \beta}} .
$$

Proof. It is easy to verify that, as $x \rightarrow \infty$,

$$
\bar{F}(x)^{-1} \sim a^{-1} \beta^{b}\left(\log \bar{G}^{-1}(x)\right)^{-b} \bar{G}(x)^{-\delta / \beta},
$$

and, therefore, we can choose

$$
\Phi(x)=a^{-1} \beta^{b}(\log x)^{-b} x^{\delta / \beta},
$$

which, by using Theorem 1, completes the proof.

The following corollary specializes $\bar{F}$ and $\bar{G}$ to have normal-like distributions, i.e. much lighter tails than exponential distributions, as shown in Corollary 1 of [8] (see also Corollary 2.2 of [1]).

Corollary 2. Suppose that $\bar{G}(x)=\mathbb{P}\left[\left|N\left(0, \sigma_{A}^{2}\right)\right|>x\right]$ and $\bar{F}(x)=\mathbb{P}\left[\left|N\left(0, \sigma_{L}^{2}\right)\right|>x\right]$, where $N\left(0, \sigma^{2}\right)$ is a Gaussian random variable with mean 0 and variance $\sigma^{2}$. Then

$$
\mathbb{P}[N>n] \sim \Gamma(\alpha+1) \alpha^{-1 / 2} \frac{(\pi \log n)^{(\alpha-1) / 2}}{n^{\alpha}},
$$

where $\alpha=\sigma_{A}^{2} / \sigma_{L}^{2}$. 
Proof. First, note that

$$
\mathbb{P}\left[\left|N\left(0, \sigma^{2}\right)\right|>x\right] \sim \frac{2 \sigma}{\sqrt{2 \pi} x} \mathrm{e}^{-x^{2} / 2 \sigma^{2}},
$$

and, therefore, recalling that $\alpha=\sigma_{A}{ }^{2} / \sigma_{L}^{2}$, we obtain

$$
\bar{F}(x) \sim \pi^{(\alpha-1) / 2} \alpha^{-1 / 2}(-\log \bar{G}(x))^{(\alpha-1) / 2}(\bar{G}(x))^{\alpha} .
$$

Hence, $\bar{F}(x)$ and $\bar{G}(x)$ satisfy the assumption of Theorem 1 with

$$
\Phi(x)=\alpha^{1 / 2}(\pi \log x)^{(1-\alpha) / 2} x^{\alpha},
$$

which implies (7).

Next, we present the proofs of Propositions 3 and 4, and Theorem 1. Note that the following proof represents a basis for the other proofs in this paper.

Proof of Proposition 3. Note that the number of retransmissions is geometrically distributed given the packet size $L$ :

$$
\mathbb{P}[N>n \mid L]=(1-\bar{G}(L))^{n}
$$

Therefore,

$$
\mathbb{P}[N>n]=\mathbb{E}\left[(1-\bar{G}(L))^{n}\right] .
$$

Since $\Phi(x)$ is eventually nondecreasing, there exists $x_{0}$ such that, for all $x>x_{0}, \Phi(x)$ has an inverse function $\Phi^{\leftarrow}(x)$. Condition (4) implies that, for $0<\varepsilon<1$, there exists $x_{\varepsilon}$ such that, for $x>x_{\varepsilon}$,

$$
(1-\varepsilon) \bar{F}(x)^{-1} \leq \Phi\left(\bar{G}(x)^{-1}\right) \leq(1+\varepsilon) \bar{F}(x)^{-1},
$$

and, thus, by choosing $x_{\varepsilon}>x_{0}$, we obtain, for $x>x_{\varepsilon}$,

$$
\Phi^{\leftarrow}\left((1-\varepsilon) \bar{F}(x)^{-1}\right) \leq \bar{G}(x)^{-1} \leq \Phi^{\leftarrow}\left((1+\varepsilon) \bar{F}(x)^{-1}\right) .
$$

We first prove the upper bound. Recalling (8), noting that $V:=\bar{F}(L)$ is a uniform random variable on $(0,1)$ (see, e.g. Proposition 2.1 of [12, Chapter 10]), and using (9), we obtain, for large $n$,

$$
\begin{aligned}
& \mathbb{P}[N>n]=\mathbb{E}\left[(1-\bar{G}(L))^{n}\right] \\
& =\mathbb{E}\left[(1-\bar{G}(L))^{n} \mathbf{1}\left(L>x_{\varepsilon}\right)\right]+\mathbb{E}\left[(1-\bar{G}(L))^{n} \mathbf{1}\left(L \leq x_{\varepsilon}\right)\right] \\
& \leq \mathbb{E}\left[\mathrm{e}^{-n / \Phi^{\leftarrow}\left((1+\varepsilon) V^{-1}\right)}\right]+\left(1-\bar{G}\left(x_{\varepsilon}\right)\right)^{n} \\
& \leq \mathbb{P}\left[0 \leq \frac{n}{\Phi^{\leftarrow}\left((1+\varepsilon) V^{-1}\right)} \leq 1\right] \\
& +\sum_{k=0}^{\lceil\log (\varepsilon n)\rceil} \mathrm{e}^{-\mathrm{e}^{k}} \mathbb{P}\left[\mathrm{e}^{k} \leq \frac{n}{\Phi^{\leftarrow}\left((1+\varepsilon) V^{-1}\right)} \leq \mathrm{e}^{k+1}\right]
\end{aligned}
$$

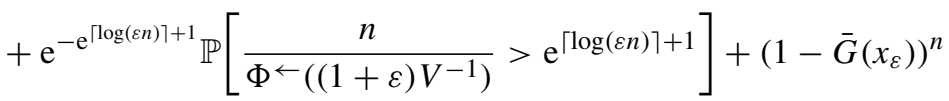

$$
\begin{aligned}
& \leq \frac{1+\varepsilon}{\Phi(n)}+\sum_{k=0}^{\lceil\log (\varepsilon n)\rceil} \mathrm{e}^{-\mathrm{e}^{k}} \frac{1+\varepsilon}{\Phi\left(n / \mathrm{e}^{k+1}\right)}+\mathrm{e}^{-\varepsilon n}+\left(1-\bar{G}\left(x_{\varepsilon}\right)\right)^{n} \text {. }
\end{aligned}
$$


Condition (2) implies that there exist finite $n_{d}$ and $d$ such that, for $n>n_{d}$,

$$
\frac{\Phi(n)}{\Phi(n / \mathrm{e})}<d
$$

resulting in, for small enough $\varepsilon$ and all $k$ satisfying $n / \mathrm{e}^{k-1} \geq 1 / \varepsilon>n_{d}$,

$$
\frac{\Phi(n)}{\Phi\left(n / \mathrm{e}^{k+1}\right)} \leq d^{k+1}
$$

and, therefore,

$$
\Phi(n) \leq \Phi\left(n_{d}\right) d^{\log \left(n / n_{d}\right)+1} .
$$

The preceding inequalities, in conjunction with (10), yield

$$
\begin{aligned}
\varlimsup_{n \rightarrow \infty} \mathbb{P}[N>n] \Phi(n) \leq & 1+\varepsilon+\sum_{k=0}^{\infty}(1+\varepsilon) \mathrm{e}^{-\mathrm{e}^{k}} d^{k+1} \\
& +\varlimsup_{n \rightarrow \infty}\left(\mathrm{e}^{-\varepsilon n}+\left(1-\bar{G}\left(x_{\varepsilon}\right)\right)^{n}\right) \Phi\left(n_{d}\right) d^{\log \left(n / n_{d}\right)+1} \\
= & 1+\varepsilon+\sum_{k=0}^{\infty}(1+\varepsilon) \mathrm{e}^{-\mathrm{e}^{k}} d^{k+1} \\
< & \infty
\end{aligned}
$$

Let us now prove the lower bound. Recalling (9) and choosing $n>x_{\varepsilon}$, we obtain

$$
\begin{aligned}
\mathbb{P}[N>n] & =\mathbb{E}\left[(1-\bar{G}(L))^{n}\right] \\
& \geq\left(1-\frac{1}{n}\right)^{n} \mathbb{P}\left[\bar{G}(L) \leq \frac{1}{n}\right] \\
& \geq\left(1-\frac{1}{n}\right)^{n} \mathbb{P}\left[\Phi^{\leftarrow}\left((1-\varepsilon) \bar{F}^{-1}(L)\right) \geq n\right] \\
& \geq\left(1-\frac{1}{n}\right)^{n} \frac{1-\varepsilon}{\Phi(n)},
\end{aligned}
$$

implying that

$$
\varliminf_{n \rightarrow \infty} \mathbb{P}[N>n] \Phi(n) \geq \lim _{n \rightarrow \infty}\left(1-\frac{1}{n}\right)^{n}(1-\varepsilon)=\mathrm{e}^{-1}(1-\varepsilon),
$$

which, in conjunction with (12), proves the proposition.

Proof of Proposition 4. Recalling (9) and choosing $n$ large enough such that $\{\bar{G}(L) \leq$ $\mathrm{e} / n\} \subseteq\left\{L>x_{\varepsilon}\right\}$ with $x_{\varepsilon}$ being the same as in (9), we obtain

$$
\begin{aligned}
\mathbb{P}[N>n] & =\mathbb{E}\left[(1-\bar{G}(L))^{n}\right] \\
& \geq\left(1-\frac{c}{n}\right)^{n} \mathbb{P}\left[\bar{G}(L) \leq \frac{c}{n}\right] \\
& \geq\left(1-\frac{c}{n}\right)^{n} \mathbb{P}\left[\Phi^{\leftarrow}\left((1-\varepsilon) \bar{F}^{-1}(L)\right) \geq \frac{n}{c}\right] \\
& \geq\left(1-\frac{c}{n}\right)^{n} \frac{1-\varepsilon}{\Phi(n / c)},
\end{aligned}
$$


implying that

$$
\varliminf_{n \rightarrow \infty} \mathbb{P}[N>n] \Phi(n) \geq \varliminf_{n \rightarrow \infty}\left(1-\frac{1}{n}\right)^{n} \frac{(1-\varepsilon) \Phi(n)}{\Phi(n / c)}=\infty,
$$

which completes the proof.

Proof of Theorem 1. We begin by proving (5). Without loss of generality, we can assume that $\Phi(x)$ is eventually absolutely continuous and strictly monotone since, by Proposition 1.5.8 of [3], we can always find an absolutely continuous and strictly monotone function

$$
\Phi^{*}(x)=\alpha \int_{x_{0}}^{x} \Phi(s) s^{-1} \mathrm{~d} s, \quad x \geq x_{0},
$$

which satisfies, as $x \rightarrow \infty$,

$$
\bar{F}(x)^{-1} \sim \Phi\left(\bar{G}(x)^{-1}\right) \sim \Phi^{*}\left(\bar{G}(x)^{-1}\right) .
$$

We first prove the upper bound. Recalling (8), noting that $V:=\bar{F}(L)$ is a uniform random variable on $(0,1)$, and using $(9)$, we obtain, for $0<\varepsilon<1$,

$$
\begin{aligned}
\mathbb{P}[N>n] & =\mathbb{E}\left[(1-\bar{G}(L))^{n}\right] \\
& =\mathbb{E}\left[(1-\bar{G}(L))^{n} \mathbf{1}\left(L \geq x_{\varepsilon}\right)\right]+\mathbb{E}\left[(1-\bar{G}(L))^{n} \mathbf{1}\left(L<x_{\varepsilon}\right)\right] \\
& \leq \mathbb{E}\left[\mathrm{e}^{-n / \Phi^{\leftarrow}\left((1+\varepsilon) V^{-1}\right)}\right]+\left(1-\bar{G}\left(x_{\varepsilon}\right)\right)^{n} .
\end{aligned}
$$

Then, by choosing integer $n_{d}$, as in (11), and noting that $\Phi(n)$ is regularly varying, the preceding inequality yields, for large $n$ and $x>x_{\varepsilon}$,

$$
\begin{aligned}
\mathbb{P}[N>n] \leq & \mathbb{E}\left[\mathrm{e}^{-n / \Phi^{\leftarrow}\left((1+\varepsilon) V^{-1}\right)} \mathbf{1}\left(0<\frac{n}{\Phi^{\leftarrow}\left((1+\varepsilon) V^{-1}\right)} \leq \mathrm{e}^{m}\right)\right] \\
& +\sum_{k=m}^{\log \left(n / n_{d}\right)-1} \mathrm{e}^{-\mathrm{e}^{k}} \mathbb{P}\left[\mathrm{e}^{k} \leq \frac{n}{\Phi^{\leftarrow}\left((1+\varepsilon) V^{-1}\right)} \leq \mathrm{e}^{k+1}\right]+O\left(\mathrm{e}^{-n / n_{d}}\right) \\
& +\left(1-\bar{G}\left(x_{\varepsilon}\right)\right)^{n} \\
\leq & \int_{\varepsilon}^{\mathrm{e}^{m}} \mathrm{e}^{-z}\left(\frac{\Phi^{\prime}(n / z)}{\Phi^{2}(n / z)} \frac{(1+\varepsilon) n}{z^{2}}\right) \mathrm{d} z+\mathbb{P}\left[\frac{n}{\Phi^{\leftarrow}\left((1+\varepsilon) V^{-1}\right)}<\varepsilon\right] \\
& +\sum_{k=m}^{\log \left(n / n_{d}\right)-1} \mathrm{e}^{-\mathrm{e}^{k}} \frac{1+\varepsilon}{\Phi\left(n / \mathrm{e}^{k+1}\right)}+o\left(\frac{1}{\Phi(n)}\right),
\end{aligned}
$$

resulting in

$$
\begin{aligned}
\mathbb{P}[N>n] \Phi(n) \leq & \int_{\varepsilon}^{\mathrm{e}^{m}} \frac{\Phi(n)}{\Phi(n / z)} \frac{\Phi^{\prime}(n / z)}{\Phi(n / z)} \frac{\mathrm{e}^{-z}(1+\varepsilon) n}{z^{2}} \mathrm{~d} z+\sum_{k=m}^{\log \left(n / n_{d}\right)-1}(1+\varepsilon) \mathrm{e}^{-\mathrm{e}^{k}} \frac{\Phi(n)}{\Phi\left(n / \mathrm{e}^{k+1}\right)} \\
& +\Phi(n) \mathbb{P}\left[\frac{n}{\Phi \leftarrow\left((1+\varepsilon) V^{-1}\right)}<\varepsilon\right]+o(1) \\
= & : I_{1}+I_{2}+I_{3}+o(1) .
\end{aligned}
$$


Since regularly varying functions are also dominantly regularly varying, the bound in (11) implies that

$$
I_{2} \leq \sum_{k=m}^{\log \left(n / n_{d}\right)-1}(1+\varepsilon) \mathrm{e}^{-\mathrm{e}^{k}} d^{k+1} \leq \sum_{k=m}^{\infty}(1+\varepsilon) \mathrm{e}^{-\mathrm{e}^{k}} d^{k+1} \rightarrow 0 \quad \text { as } m \rightarrow \infty .
$$

For $I_{1}$, since $\Phi(n)$ is regularly varying, by the uniform convergence theorem of slowly varying functions (see Theorem 1.2.1 of [3]), it is easy to obtain uniformly for $\varepsilon \leq z \leq \mathrm{e}^{m}$, as $n \rightarrow \infty$,

$$
\frac{\Phi(n)}{\Phi(n / z)} \sim z^{\alpha}
$$

and, recalling (13),

$$
\frac{\Phi^{\prime}(n / z)}{\Phi(n / z)}=\frac{z \alpha}{n}
$$

which imply that

$$
I_{1} \sim \int_{\varepsilon}^{\mathrm{e}^{m}}(1+\varepsilon) \alpha \mathrm{e}^{-z} z^{\alpha-1} \mathrm{~d} z
$$

Furthermore, $\Phi(n)$ being regularly varying $(\alpha>0)$ implies that $I_{3} \rightarrow 0$ as passing $n \rightarrow \infty$ and then $\varepsilon \rightarrow 0$. Thus, passing $n \rightarrow \infty$ in (14), recalling (15), and then passing $m \rightarrow \infty$ and $\varepsilon \rightarrow 0$, we obtain

$$
\mathbb{P}[N>n] \Phi(n) \lesssim \int_{0}^{\infty} \alpha \mathrm{e}^{-z} z^{\alpha-1} \mathrm{~d} z=\Gamma(\alpha+1) .
$$

For the lower bound, the proof follows similar arguments, and the details are presented in Subsection 3.2. The same subsection also contains the proof of the statement (ii) of the theorem.

Informally, the condition of $\Phi(\cdot)$ being dominantly varying is almost necessary in order for $\mathbb{P}[N>n] \approx \Phi(n)^{-1}$ to hold. As shown in Proposition 5, this tail equivalence does not hold if $\Phi(\cdot)$ is not dominantly varying, e.g. $\Phi(n)=\mathrm{e}^{(\log n)^{2}}$ (lognormal type). Here, we further characterize the behavior of the lognormal-type distributions in the following proposition.

Proposition 5. If $\log (\Phi(x))=\lambda(\log x)^{\delta}, \delta>1$ and $\lambda>0$, then, under condition (4), we obtain

$$
\lim _{n \rightarrow \infty} \frac{\log \left(\mathbb{P}[N>n]^{-1}\right)-\log (\Phi(n))}{(\log \log n)(\log n)^{\delta-1}}=-\lambda \delta(\delta-1) .
$$

The proof of this proposition is presented in Subsection 3.3.

Remark 5. In Proposition 5, it can be verified that $\Phi(\cdot)$ is not dominantly regularly varying. Therefore, by Propositions 3 and 4, we know that $\mathbb{P}[N>n] \Phi(n) \rightarrow \infty$ as $n \rightarrow \infty$. However, Proposition 5 further characterizes how fast $\mathbb{P}[N>n] \Phi(n)$ goes to infinity in the logarithmic scale, which also implies the weaker result

$$
\lim _{n \rightarrow \infty} \frac{\log \left(\mathbb{P}[N>n]^{-1}\right)}{\log (\Phi(n))}=1 .
$$

In the following theorem we extend the preceding logarithmic limit under a more general condition on $\Phi(\cdot)$. 
Theorem 2. If $\Phi(x)=\mathrm{e}^{l(x)}$ satisfies (3), where $l(x)$ is slowly varying with

$$
\lim _{x \rightarrow \infty} \frac{l(x / l(x))}{l(x)}=1
$$

then

$$
\lim _{n \rightarrow \infty} \frac{\log \left(\mathbb{P}[N>n]^{-1}\right)}{\log \Phi(n)}=1 .
$$

Remark 6. It can be easily verified that if $\log (\Phi(x))=\mathrm{e}^{(\log x)^{\delta}}$ then condition (18) holds when $0<\delta<\frac{1}{2}$ and does not hold when $\delta \geq \frac{1}{2}$. Furthermore, if $\log (\Phi(x))=\Psi(\log x)$, where $\Psi(x)$ is regularly varying, e.g. $\Phi(x)^{-1}$ being lognormal, then condition (18) also holds; we state this result as Corollary 3 below.

Remark 7. (Lattice variables.) When $L$ is lattice valued, it is easy to see from the proof of Theorem 2 that, if there exists a continuous random variable $L^{*}$ such that $\log \mathbb{P}\left[L^{*}>x\right] \sim$ $\log \mathbb{P}[L>x]$ as $x \rightarrow \infty$, or, equivalently, if there exists a continuous negative nonincreasing function $q(x)$ such that $\log \mathbb{P}[L>x] \sim q(x)$, then Theorem 2 still holds, e.g. when $L$ has a geometric or Poisson distribution. To rigorously prove this claim, we can use similar arguments as in the proof of Theorem 3.1 of [9]. Note that this remark also applies to other logarithmic asymptotics; see, e.g. Corollary 3, Propositions 6 and 7, and Theorems 3, 4, 6, and 7.

Corollary 3. If a regularly varying function $\Psi(\cdot)$ with a nonnegative index satisfies

$$
\lim _{x \rightarrow \infty} \frac{\log \bar{F}(x)^{-1}}{\Psi\left(\log \bar{G}(x)^{-1}\right)}=1
$$

and, in addition, is eventually nondecreasing when $\Psi(\cdot)$ is slowly varying, then we have

$$
\lim _{n \rightarrow \infty} \frac{\log \left(\mathbb{P}[N>n]^{-1}\right)}{\Psi(\log n)}=1 .
$$

Remark 8. Corollary 3 and, more precisely, Theorem 6 in Subsection 2.2 , imply parts $(1: 1)$, (2:1), and (2:2) of Theorem 2.1 of [1] and extend Theorem 2 of [8]. The latter theorem states that if, for $\alpha>0, \lim _{x \rightarrow \infty} \log \bar{F}(x) / \log \bar{G}(x)=\alpha$ then $\lim _{n \rightarrow \infty} \log \mathbb{P}[N>n] / \log n=-\alpha$.

Proof of Corollary 3. For a regularly varying function $\Psi(\cdot)$, it is easy to verify that $l(x)=$ $\Psi(\log (x))$ satisfies

$$
\lim _{x \rightarrow \infty} \frac{l(x / l(x))}{l(x)}=\lim _{x \rightarrow \infty} \frac{\Psi(\log x-\log \Psi(\log (x)))}{\Psi(\log (x))}=1,
$$

and, therefore, by Theorem 2, we prove the corollary.

Remark 9. Note that, in conjunction with Remark 6, condition (18) is close to necessary since (19) does not hold if $\log (\Phi(x))=\mathrm{e}^{(\log x)^{\delta}}, \frac{1}{2}<\delta<1$, as can be seen from the following proposition.

Proposition 6. If $\log (\Phi(x))=\mathrm{e}^{\lambda(\log x)^{\delta}}, \frac{1}{2}<\delta<1$ and $\lambda>0$, then, under condition (3), we obtain

$$
\log \left(\log \left(\mathbb{P}[N>n]^{-1}\right)\right)-\log (\log (\Phi(n))) \sim-\delta \lambda^{2}(\log n)^{2 \delta-1} .
$$

The proof of this proposition is presented in Subsection 3.4. 
Remark 10. Note that Proposition 6 implies that, for $0<\varepsilon<1$ and large $n$,

$$
0 \leq \frac{\log \left(\mathbb{P}[N>n]^{-1}\right)}{\log \Phi(n)} \leq \mathrm{e}^{-(1-\varepsilon) \delta \lambda^{2}(\log n)^{2 \delta-1}} \rightarrow 0,
$$

which is not covered by the limit in (19).

Proof of Theorem 2. Since $\Phi(x)$ is eventually nondecreasing, there exists $x_{0}$ such that, for all $x>x_{0}, \Phi(x)$ has an inverse function $\Phi^{\leftarrow}(x)$. Condition (3) implies that, for $0<\varepsilon<1$, there exists $x_{\varepsilon} \geq x_{0}$, such that, for $x>x_{\varepsilon}$,

$$
\bar{F}(x)^{-(1-\varepsilon)} \leq \Phi\left(\bar{G}(x)^{-1}\right) \leq \bar{F}(x)^{-(1+\varepsilon)} .
$$

Thus, for $x>x_{\varepsilon}$, we obtain

$$
\Phi^{\leftarrow}\left(\bar{F}(x)^{-(1-\varepsilon)}\right) \leq \bar{G}(x)^{-1} \leq \Phi^{\leftarrow}\left(\bar{F}(x)^{-(1+\varepsilon)}\right) .
$$

We first prove the upper bound. Recalling (8), noting that $V:=\bar{F}(L)$ is a uniform random variable on $(0,1)$, and using (20), we obtain, for integer $y$ and large $n$,

$$
\begin{aligned}
\mathbb{P}[N>n] & =\mathbb{E}\left[(1-\bar{G}(L))^{n}\right] \\
& =\mathbb{E}\left[(1-\bar{G}(L))^{n} \mathbf{1}\left(L>x_{\varepsilon}\right)\right]+\mathbb{E}\left[(1-\bar{G}(L))^{n} \mathbf{1}\left(L \leq x_{\varepsilon}\right)\right] \\
& \leq \mathbb{E}\left[\mathrm{e}^{-n / \Phi^{\leftarrow}\left(V^{-(1+\varepsilon)}\right)}\right]+\left(1-\bar{G}\left(x_{\varepsilon}\right)\right)^{n} \\
& \leq \sum_{k=0}^{y} \mathrm{e}^{-k} \mathbb{P}\left[k \leq \frac{n}{\Phi^{\leftarrow}\left(V^{-(1+\varepsilon)}\right)} \leq k+1\right]+\mathrm{e}^{-(y+1)}+\left(1-\bar{G}\left(x_{\varepsilon}\right)\right)^{n},
\end{aligned}
$$

which, using the fact that $\mathbb{P}[N>n]$ decays slower than any exponential function (see Proposition 1), noting that $\Phi(x)=\mathrm{e}^{l(x)}$, and choosing $y=\lceil l(n)\rceil-1$, implies that

$$
\begin{aligned}
\mathbb{P}[N>n] & \leq \sum_{k=0}^{\lceil l(n)\rceil-1} \exp \left(-k-\frac{1}{1+\varepsilon} l\left(\frac{n}{k+1}\right)\right)+\mathrm{e}^{-l(n)}+o(\mathbb{P}[N>n]) \\
& \leq\lceil l(n)\rceil \exp \left(-\frac{1}{1+\varepsilon} l\left(\frac{n}{\lceil l(n)\rceil}\right)\right)+\mathrm{e}^{-l(n)}+o(\mathbb{P}[N>n]) .
\end{aligned}
$$

From (3), it is easy to see that $l(x)$ increases to infinity when $x \rightarrow \infty$ and, since $l(x)$ is slowly varying, by (18) and (21), we obtain

$$
\varliminf_{n \rightarrow \infty} \frac{\log \mathbb{P}[N>n]^{-1}}{l(n)} \geq 1 .
$$

Let us now prove the lower bound. Recalling (20) and choosing large enough $n$, we obtain

$$
\begin{aligned}
\mathbb{P}[N>n] & =\mathbb{E}\left[(1-\bar{G}(L))^{n}\right] \\
& \geq\left(1-\frac{1}{n}\right)^{n} \mathbb{P}\left[\bar{G}(L) \leq \frac{1}{n}\right] \\
& \geq\left(1-\frac{1}{n}\right)^{n} \mathbb{P}\left[\Phi^{\leftarrow}\left(\bar{F}^{-(1-\varepsilon)}(L)\right) \geq n\right] \\
& \geq\left(1-\frac{1}{n}\right)^{n} \frac{1}{\Phi(n)^{1 /(1-\varepsilon)}},
\end{aligned}
$$


implying that

$$
\varlimsup_{n \rightarrow \infty} \frac{\log \mathbb{P}[N>n]^{-1}}{l(n)} \leq \frac{1}{1-\varepsilon},
$$

which, by passing $\varepsilon \rightarrow 0$ and in conjunction with (22), proves the theorem.

2.1.2. Medium heavy (Weibull) asymptotics. In the preceding subsection, we studied the scenario when the distribution of $N$ is heavier than any Weibull distribution. Specifically, we established the necessary conditions under which $\mathbb{P}[N>n] \approx \Phi^{-1}(n)$ holds when the separation between $\mathbb{P}[L>x]$ and $\mathbb{P}[A>x]$ can be characterized in the form of $\Phi(x)=\mathrm{e}^{l(x)}$ with $l(x)$ being slowly varying. In this subsection, we further increase the separation in the sense that $\Phi(x)=\mathrm{e}^{R_{\beta}(x)}$ with $R_{\beta}(x)$ being regularly varying of index $\beta>0$, and, under this condition, the distribution of $N$ is shown to be of Weibull type. In this situation, the tail equivalence developed in the preceding subsection does not hold anymore and admits a different form, as stated in the following theorem.

Theorem 3. If $\Phi(x)=\mathrm{e}^{R_{\beta}(x)}$ satisfies (3), where $R_{\beta}(x)=x^{\beta} l(x), \beta>0$, is regularlyvarying with $l(x)$ satisfying

$$
\lim _{x \rightarrow \infty} \frac{l\left((x / l(x))^{1 /(1+\beta)}\right)}{l(x)}=1,
$$

then

$$
\lim _{n \rightarrow \infty} \frac{\log \left(\mathbb{P}[N>n]^{-1}\right)}{(\log \Phi(n))^{1 /(\beta+1)}}=\beta^{1 /(\beta+1)}+\beta^{-\beta /(\beta+1)} .
$$

Remark 11. Theorem 3 and, more precisely, Theorem 7 in Subsection 2.2, imply part (1:2) of Theorem 2.1 of [1], and provide a more precise logarithmic asymptotic instead of a double logarithmic limit that was proved in [1]. Furthermore, although condition (23) appears complicated, it is easy to check that any slowly varying function $l(x)=l_{1}(\log x)$ satisfies it, where $l_{1}(\cdot)$ is also a slowly varying function.

Proof of Theorem 3. We first begin by proving the upper bound. Following the same approach as in the proof of Theorem 2, we obtain, for $\varepsilon>0$, integer $y$, and large enough $n$,

$$
\begin{aligned}
\mathbb{P}[N>n] & \leq \sum_{k=0}^{y-1} \mathrm{e}^{-k} \mathbb{P}\left[k \leq \frac{n}{\Phi^{\leftarrow}\left(V^{-(1+\varepsilon)}\right)} \leq k+1\right]+\mathrm{e}^{-y}+o(\mathbb{P}[N>n]) \\
& \leq \sum_{k=0}^{y-1} \exp \left(-k-\frac{1}{1+\varepsilon} R_{\beta}\left(\frac{n}{k+1}\right)\right)+\mathrm{e}^{-y}+o(\mathbb{P}[N>n]) .
\end{aligned}
$$

Using the same argument as in (13), we can find an absolutely continuous and strictly increasing function $R_{\beta}^{*}(u):=\beta \int_{u_{0}}^{u} R_{\beta}(s) s^{-1} \mathrm{~d} s, u \geq u_{0}>0$, that is a modified asymptotically equivalent version of $R_{\beta}(u)$. The newly constructed function $R_{\beta}^{*}(u)$ has the property that, for $0<\varepsilon<1$, there exists $y_{\varepsilon}>u_{0}$ such that $(1-\varepsilon) R_{\beta}^{*}(u)<R_{\beta}(u)<(1+\varepsilon) R_{\beta}^{*}(u)$ for $u>y_{\varepsilon}$. Therefore, for $0<x<n / y_{\varepsilon}$,

$$
x+\frac{1}{1+\varepsilon} R_{\beta}\left(\frac{n}{x}\right) \geq x+\frac{1-\varepsilon}{1+\varepsilon} R_{\beta}^{*}\left(\frac{n}{x}\right),
$$

and, for $u \geq u_{0}$

$$
\left(R_{\beta}^{*}(u)\right)^{\prime}=\beta u^{\beta-1} l(u)
$$


Choosing $y=\left\lceil n / y_{\varepsilon}\right\rceil$ in (25) and using the asymptotic equivalence relationship between $R_{\beta}(\cdot)$ and $R_{\beta}^{*}(\cdot)$, we obtain

$$
\begin{aligned}
\mathbb{P}[N>n] & \leq \sum_{k=0}^{\left\lceil n / y_{\varepsilon}\right\rceil-1} \exp \left(-k-\frac{1}{1+\varepsilon} R_{\beta}\left(\frac{n}{k+1}\right)\right)+\mathrm{e}^{-n / y_{\varepsilon}}+o(\mathbb{P}[N>n]) \\
& \leq \sum_{k=0}^{\left\lceil n / y_{\varepsilon}\right\rceil-1} \exp \left(-k-\frac{1-\varepsilon}{1+\varepsilon} R_{\beta}^{*}\left(\frac{n}{k+1}\right)\right)+o(\mathbb{P}[N>n]) .
\end{aligned}
$$

Next, let $f(x)=x+R_{\beta}^{*}(n / x)(1-\varepsilon) /(1+\varepsilon)$, and, from (26), it is easy to check that

$$
f^{\prime}(x)=1-\frac{1-\varepsilon}{1+\varepsilon}\left(R_{\beta}^{*}\left(\frac{n}{x}\right)\right)^{\prime} \frac{n}{x^{2}}=1-\frac{1-\varepsilon}{1+\varepsilon} \frac{\beta}{n} \frac{n^{\beta+1}}{x^{\beta+1}} l\left(\frac{n}{x}\right) .
$$

Then, defining $g(u):=u^{\beta+1} l(u)$, and using the same argument as in the construction of $R_{\beta}^{*}(\cdot)$, we can find an absolutely continuous and strictly increasing function

$$
g^{*}(u):=(\beta+1) \int_{u_{0}}^{u} u^{\beta} l(u) \mathrm{d} s, \quad u \geq u_{0},
$$

such that $(1-\varepsilon) g(u)<g^{*}(u)<(1+\varepsilon) g(u), u>y_{\varepsilon}$; possibly for a larger $y_{\varepsilon}$. Therefore, for $0<x<n / y_{\varepsilon}$, we obtain

$$
1-\frac{1}{1+\varepsilon} \frac{\beta}{n} g^{*}\left(\frac{n}{x}\right)<f^{\prime}(x)=1-\frac{1-\varepsilon}{1+\varepsilon} \frac{\beta}{n} g\left(\frac{n}{x}\right)<1-\frac{1-\varepsilon}{(1+\varepsilon)^{2}} \frac{\beta}{n} g^{*}\left(\frac{n}{x}\right),
$$

where, as shown in the preceding inequalities, the lower and upper bounds of $f^{\prime}(x)$ are two monotonically increasing functions for $0<x<n / y_{\varepsilon}$.

Now, define

$$
x_{1}:=\left(\frac{(1-\varepsilon)^{3}}{(1+\varepsilon)^{2}}\right)^{1 /(\beta+1)} \beta^{1 /(\beta+1)} n^{\beta /(\beta+1)} l(n)^{1 /(\beta+1)}
$$

and

$$
x_{2}:=(1+\varepsilon)^{1 /(\beta+1)} \beta^{1 /(\beta+1)} n^{\beta /(\beta+1)} l(n)^{1 /(\beta+1)} .
$$

After some straightforward calculations, by condition (23), for large enough $n$, we obtain

$$
f^{\prime}\left(x_{1}\right) \leq 1-\frac{1-\varepsilon}{(1+\varepsilon)^{2}} \frac{\beta}{n} g^{*}\left(\frac{n}{x_{1}}\right)<1-\left(\frac{1-\varepsilon}{1+\varepsilon}\right)^{2} \frac{\beta}{n} g\left(\frac{n}{x_{1}}\right)<0
$$

and

$$
f^{\prime}\left(x_{2}\right) \geq 1-\frac{1}{1+\varepsilon} \frac{\beta}{n} g^{*}\left(\frac{n}{x_{2}}\right)>1-\frac{\beta}{n} g\left(\frac{n}{x_{2}}\right)>0,
$$

which, by (28), imply that $f^{\prime}(x)<0$ for all $0<x \leq x_{1}$ and $f^{\prime}(x)>0$ for all $x_{2} \leq x<n / y_{\varepsilon}$. Therefore, for all $x \in\left(0, x_{1}\right] \cup\left[x_{2}, n / y_{\varepsilon}\right), f(x)$ is bounded from below by $\min \left(f\left(x_{1}\right), f\left(x_{2}\right)\right)$. Now,

$$
\begin{aligned}
\min \left(f\left(x_{1}\right), f\left(x_{2}\right)\right) & \geq \inf _{x \in\left[x_{1}, x_{2}\right]} f(x) \\
& \geq x_{1}+\inf _{x \in\left[x_{1}, x_{2}\right]} \frac{1-\varepsilon}{1+\varepsilon} R_{\beta}^{*}\left(\frac{n}{x}\right) \\
& \geq x_{1}+\frac{1-\varepsilon}{1+\varepsilon} R_{\beta}^{*}\left(\frac{n}{x_{2}}\right)
\end{aligned}
$$


since $R_{\beta}^{*}(u)$ is increasing for $u \geq u_{0}$. Hence, using (27) and (29), and recalling that $R_{\beta}(u)<(1+\varepsilon) R_{\beta}^{*}(u)$, we obtain

$$
\mathbb{P}[N>n] \leq\left\lceil\frac{n}{y_{\varepsilon}}\right\rceil \exp \left(1-x_{1}-\frac{1}{(1+\varepsilon)^{2}} R_{\beta}\left(\frac{n}{x_{2}}\right)\right)+o(\mathbb{P}[N>n]),
$$

resulting in

$$
\varliminf_{n \rightarrow \infty} \frac{\log \left(\mathbb{P}[N>n]^{-1}\right)}{n^{\beta /(\beta+1)} l(n)^{1 /(\beta+1)}} \geq\left(\frac{(1-\varepsilon)^{3}}{(1+\varepsilon)^{2}}\right)^{1 /(\beta+1)} \beta^{1 /(\beta+1)}+(1+\varepsilon)^{-\beta /(\beta+1)-2} \beta^{-\beta /(\beta+1)} .
$$

Passing $\varepsilon \rightarrow 0$ in the preceding inequality yields

$$
\varliminf_{n \rightarrow \infty} \frac{\log \left(\mathbb{P}[N>n]^{-1}\right)}{n^{\beta /(\beta+1)} l(n)^{1 /(\beta+1)}} \geq \beta^{1 /(\beta+1)}+\beta^{-\beta /(\beta+1)} .
$$

Let us now prove the lower bound. By recalling condition (20) and using $1-u \geq \mathrm{e}^{-(1+\varepsilon) u}$ for small enough $u$, we obtain, for $x_{n}=o(n)>0$ and large enough $n$,

$$
\begin{aligned}
\mathbb{P}[N>n] & \geq \mathbb{E}\left[(1-\bar{G}(L))^{n} \mathbf{1}\left(L \geq x_{\varepsilon}\right)\right] \\
& \geq \mathbb{E}\left[\mathrm{e}^{-(1+\varepsilon) \bar{G}(L) n} \mathbf{1}\left(L \geq x_{\varepsilon}\right)\right] \\
& \geq \mathbb{E}\left[\mathrm{e}^{-(1+\varepsilon) n / \Phi^{\leftarrow}\left(V^{-(1-\varepsilon)}\right)} \mathbf{1}\left(V \leq \bar{F}\left(x_{\varepsilon}\right)\right)\right] \\
& \geq \mathrm{e}^{-x_{n}} \mathbb{P}\left[\frac{(1+\varepsilon) n}{\Phi^{\leftarrow}\left(V^{-(1-\varepsilon)}\right)} \leq x_{n}, V \leq \bar{F}\left(x_{\varepsilon}\right)\right] \\
& =\mathrm{e}^{-x_{n}} \Phi\left(\frac{(1+\varepsilon) n}{x_{n}}\right)^{-1 /(1-\varepsilon)} \\
& =\exp \left(-x_{n}-\frac{1}{1-\varepsilon} R_{\beta}\left(\frac{(1+\varepsilon) n}{x_{n}}\right)\right),
\end{aligned}
$$

since $\left\{(1+\varepsilon) n / \Phi^{\leftarrow}\left(V^{-(1-\varepsilon)}\right) \leq x_{n}\right\}$ implies that $\left\{V \leq \bar{F}\left(x_{\varepsilon}\right)\right\}$ for all large enough $n$. Next, choosing $x_{n}=\beta^{1 /(\beta+1)} n^{\beta /(\beta+1)} l(n)^{1 /(\beta+1)}$, using condition (23), and then passing $n \rightarrow \infty$ as well as $\varepsilon \rightarrow 0$, we obtain

$$
\varlimsup_{n \rightarrow \infty} \frac{\log \left(\mathbb{P}[N>n]^{-1}\right)}{n^{\beta /(\beta+1)} l(n)^{1 /(\beta+1)}} \leq \beta^{1 /(\beta+1)}+\beta^{-\beta /(\beta+1)} .
$$

Finally, combining (30) and (31) completes the proof.

2.1.3. Nearly exponential asymptotics. In the preceding subsection, the functional separation between $\mathbb{P}[L>x]$ and $\mathbb{P}[A>x]$ was characterized in the form of $\Phi(x)=\mathrm{e}^{R_{\gamma}(x)}$ with $R_{\gamma}(x)$ being regularly varying. In this subsection we investigate the situation when the separation in terms of $\Phi(x)$ is even larger than $\mathrm{e}^{R_{\gamma}(x)}$, which leads to the nearly exponential asymptotics for $\mathbb{P}[N>n]$ in the following proposition and Theorem 4 below.

Proposition 7. If $\log \left(\bar{F}(x)^{-1}\right) \sim \mathrm{e}^{\left(\log \left(\bar{G}(x)^{-1}\right)\right)^{\delta}}, \delta>1$, then

$$
\log \left(\log \left(\mathbb{P}[N>n]^{-1}\right)\right)-\log n+(\log n)^{1 / \delta} \sim \frac{1}{\delta}(\log n)^{2 / \delta-1} .
$$


Remark 12. Observe that $\delta=2$ represents another critical point since $(\log n)^{2 / \delta-1}$ converges to 0 or $\infty$ if $\delta>2$ or $1<\delta<2$, respectively. Furthermore, the result shows that $\mathbb{P}[N>n] \approx \exp \left(-n / \mathrm{e}^{(\log n)^{1 / \delta}}\right)$, which means that $N$ is nearly exponential because $\mathrm{e}^{(\log n)^{1 / \delta}}$ is slowly varying for $\delta>1$ (see [3, p. 16]). In addition, informally speaking, we point out that $\delta=1$ corresponds to the Weibull case already covered by Theorem 3 in Subsection 2.1.2, meaning that Proposition 7 describes the change in functional behavior on the boundary between the Weibull case and the nearly exponential case.

Proof of Proposition 7. We first prove the upper bound. Following the same approach as in the proof of Theorem 2, we obtain, for $\varepsilon>0$,

$$
\mathbb{P}[N>n] \leq \sum_{k=0}^{n-1} \exp \left(-k-\frac{1}{1+\varepsilon} \mathrm{e}^{(\log n-\log (k+1))^{\delta}}\right)+o(\mathbb{P}[N>n]) .
$$

Then, we bound the preceding sum by estimating the minimum of

$$
f(x):=x+\frac{1}{1+\varepsilon} \mathrm{e}^{(\log n-\log x)^{\delta}} .
$$

We can check that $f^{\prime}(x)$ is an increasing function and $f(x)$ is convex in $x$ on $(0, n]$. Now, for $0<\varepsilon<1$, define

$$
x_{1}:=\frac{n}{\mathrm{e}^{\left(\log n-(1-\varepsilon)(\log n)^{1 / \delta}\right)^{1 / \delta}}},
$$

and, for large enough $n$, it can be verified that $f^{\prime}\left(x_{1}\right)<0$, implying that $f(x)^{\prime}<0$ for $x<x_{1}$. Therefore, the point $x^{*}$ where $f(x)$ achieves its minimum on $(0, n]$ satisfies

$$
x^{*} \geq x_{1}
$$

Combining (32) and (33), we obtain, for large $n$,

$$
\mathbb{P}[N>n] \leq n \mathrm{e}^{1-f\left(x^{*}\right)}+o(\mathbb{P}[N>n]) \leq n \mathrm{e}^{1-x_{1}}+o(\mathbb{P}[N>n])<2 n \mathrm{e}^{1-x_{1}},
$$

and, therefore, for large enough $n$,

$$
\log \left(\mathbb{P}[N>n]^{-1}\right) \geq \frac{n}{\mathrm{e}^{\left(\log n-(1-\varepsilon)(\log n)^{1 / \delta}\right)^{1 / \delta}}}-\log (2 n)-1,
$$

which implies that

$$
\log \left(\log \left(\mathbb{P}[N>n]^{-1}\right)\right)-\log n+(\log n)^{1 / \delta} \gtrsim \frac{1}{\delta}(\log n)^{2 / \delta-1} .
$$

Let us now prove the lower bound. By using the same arguments as in the proof of the lower bound for Theorem 3, we obtain, for large enough $n$,

$$
\log \left(\mathbb{P}[N>n]^{-1}\right) \leq x_{n}+\frac{1}{1-\varepsilon} \exp \left(\left(\log \left(\frac{(1+\varepsilon) n}{x_{n}}\right)\right)^{\delta}\right)
$$

which, by choosing $x_{n}=(1+\varepsilon) n \mathrm{e}^{-\left(\log n-(\log n)^{1 / \delta}\right)^{1 / \delta}}$, and passing $n \rightarrow \infty$ and then $\varepsilon \rightarrow 0$, yields

$$
\log \left(\log \left(\mathbb{P}[N>n]^{-1}\right)\right)-\log n+(\log n)^{1 / \delta} \lesssim \frac{1}{\delta}(\log n)^{2 / \delta-1} .
$$

Finally, combining (34) and (35) completes the proof. 
Theorem 4. If $\log \left(\bar{F}(x)^{-1}\right) \sim \mathrm{e}^{R_{\gamma}\left(\bar{G}(x)^{-1}\right)}$, where $R_{\gamma}(\cdot)$ is positive, locally bounded on $[0, \infty)$, and regularly varying with index $\gamma>0$, then

$$
\log \mathbb{P}[N>n]^{-1} \sim \frac{n}{R_{\gamma}^{\leftarrow}(\log n)}
$$

where $R_{\gamma}^{\leftarrow}(\cdot)$ is the generalized inverse of $R_{\gamma}(\cdot)$.

Remark 13. Note that the functional form in (36) is different from that in (24) which describes the Weibull case. In principle, one could study the situations where $\Phi(\cdot)$ grows faster than three exponential scales, which would make the distributions of $N$ even closer to the exponential one. However, from a practical point of view, these cases will basically be indistinguishable from the exponential distribution and, thus, we omit these derivations.

Proof of Theorem 4. We first prove the upper bound. Following the same approach as in the proof of Theorem 2, we obtain, for $0<\varepsilon<1$ and $y>0$,

$$
\mathbb{P}[N>n] \leq \sum_{k=0}^{\lfloor n / y\rfloor-1} \exp \left(-k-\frac{1}{1+\varepsilon} \mathrm{e}^{R_{\gamma}(n /(k+1))}\right)+o(\mathbb{P}[N>n]) .
$$

By using the same argument as in (13), we can choose $R_{\gamma}^{*}(x)=\gamma \int_{1}^{x} R_{\gamma}(s) s^{-1} \mathrm{~d} s, x \geq 1$, and observe that $R_{\gamma}^{*}(\cdot)$ is absolutely continuous, strictly increasing with an inverse $R_{\gamma}^{* \leftarrow}(\cdot)$.

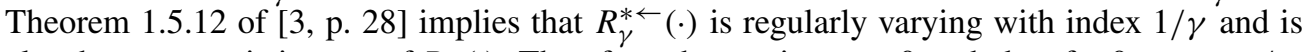
also the asymptotic inverse of $R_{\gamma}(\cdot)$. Therefore, there exists $y>0$ such that, for $0<x<n / y$,

$$
x+\frac{1}{1+\varepsilon} \mathrm{e}^{R_{\gamma}(n / x)} \geq x+\frac{1}{1+\varepsilon} \mathrm{e}^{(1-\varepsilon) R_{\gamma}^{*}(n / x)} .
$$

Now, define $f(x):=x+\mathrm{e}^{(1-\varepsilon) R_{\gamma}^{*}(n / x)} /(1+\varepsilon)$, and note that

$$
\begin{aligned}
f^{\prime}(x) & =1-\frac{1-\varepsilon}{1+\varepsilon} \mathrm{e}^{(1-\varepsilon) R_{\gamma}^{*}(n / x)}\left(R_{\gamma}^{*}\left(\frac{n}{x}\right)\right)^{\prime} \frac{n}{x^{2}} \\
& =1-\frac{1-\varepsilon}{1+\varepsilon} \mathrm{e}^{(1-\varepsilon) R_{\gamma}^{*}(n / x)} \frac{\gamma R_{\gamma}(n / x)}{x} \\
& \leq 1-\frac{1-\varepsilon}{1+\varepsilon} \mathrm{e}^{(1-\varepsilon)^{2} R_{\gamma}^{*}(n / x)} \frac{\gamma R_{\gamma}^{*}(n / x)}{x},
\end{aligned}
$$

where the right-hand side of the above inequality is an increasing function in $x$ on $(0, n / y)$ for large enough $y$. Next, defining

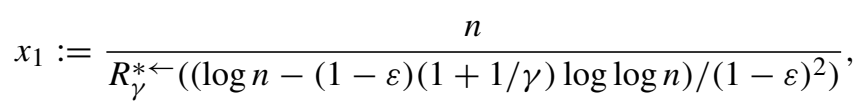

it can be verified that, for all large enough $n, f^{\prime}\left(x_{1}\right)$ is bounded from above by

$$
1-\frac{\gamma R_{\gamma}^{* \leftarrow}\left((\log n-(1-\varepsilon)(1+1 / \gamma) \log \log n) /(1-\varepsilon)^{2}\right)(\log n-(1-\varepsilon)(1+1 / \gamma) \log \log n)}{\left(1-\varepsilon^{2}\right)(\log n)^{(1-\varepsilon)(1+1 / \gamma)}}<0,
$$

which implies that $f(x)^{\prime}<0$ for $0<x \leq x_{1}$ and large $n$. Thus, $\inf _{x \in\left(0, x_{1}\right]} f(x) \geq f\left(x_{1}\right) \geq x_{1}$; observe that inf $\operatorname{se}_{x \in\left(x_{1}, n / y\right)} f(x) \geq \inf _{x \in\left(x_{1}, n / y\right)} x \geq x_{1}$, implying that

$$
\inf _{x \in(0, n / y)} f(x) \geq f\left(x_{1}\right) \geq x_{1} .
$$


Combining (37) and (38) yields, for large enough $n$,

$$
\mathbb{P}[N>n] \leq \frac{n}{y} \mathrm{e}^{1-f\left(x_{1}\right)}+o(\mathbb{P}[N>n]) \leq \frac{2 n}{y} \mathrm{e}^{1-x_{1}},
$$

resulting in

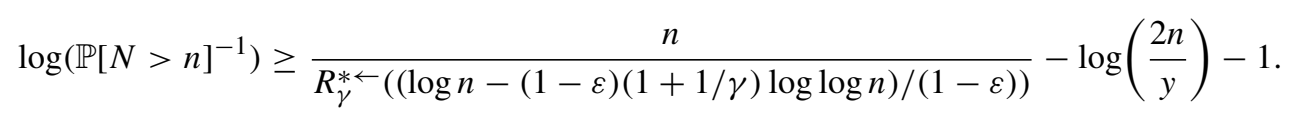

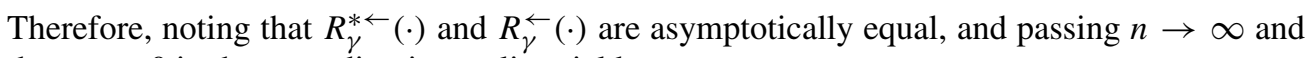
then $\varepsilon \rightarrow 0$ in the preceding inequality yields

$$
\log \mathbb{P}[N>n]^{-1} \gtrsim \frac{n}{R_{\gamma}^{\leftarrow}(\log n)}
$$

Let us now prove the lower bound. By using the same arguments as in the proof of the lower bound for Theorem 3, we obtain, for large enough $n$,

$$
\log \left(\mathbb{P}[N>n]^{-1}\right) \leq x_{n}+\frac{1}{1-\varepsilon} \mathrm{e}^{R_{\gamma}\left((1+\varepsilon) n / x_{n}\right)},
$$

which, by choosing

$$
x_{n}=\frac{(1+\varepsilon) n}{R_{\gamma}^{\leftarrow}((1-\varepsilon) \log n-(1 / \gamma) \log \log n)}
$$

and noting that $R_{\gamma}\left(R_{\gamma}^{\leftarrow}(u)\right) \leq u /(1-\varepsilon)$ for all large enough $u$, yields, for large $n$,

$$
\log \left(\mathbb{P}[N>n]^{-1}\right) \leq x_{n}+\frac{n}{(1-\varepsilon)(\log n)^{1 /(1-\varepsilon) \gamma}} .
$$

The above inequality implies that

$$
\log \left(\mathbb{P}[N>n]^{-1}\right) \lesssim \frac{n}{R_{\gamma}^{\leftarrow}(\log n)}
$$

Finally, combining (39) and (40) completes the proof.

\subsection{Asymptotics of the total transmission time $T$}

In this subsection we compute the asymptotics of the total transmission time $T$ based on the previous results on $\mathbb{P}[N>n]$ and the relationship between $N$ and $T$ described in (1). Theorem 5 and Theorem 6 below characterize the exact asymptotics and logarithmic asymptotics for the very heavy case, respectively, and Theorem 7 below gives the result for the moderate heavy (Weibull) case. Interestingly, we want to point out that the minimum conditions needed for Theorem 7, as shown by Proposition 8 below, involve an intriguing balance between the tail decays of $\mathbb{P}[A>x]$ and $\mathbb{P}[L>x]$.

Similarly, the corresponding results on $T$ can be derived for the other statements on $N$, e.g. Propositions 3, 4, 5, and 6, and Theorem 4. However, to avoid lengthy expositions and repetitions, we omit these derivations. In the following, let $\vee \equiv \max$.

Theorem 5. If $\mathbb{E}\left[U^{(\alpha \vee 1)+\theta}\right]<\infty, \mathbb{E}\left[A^{1+\theta}\right]<\infty$, and $\mathbb{E}\left[L^{\alpha+\theta}\right]<\infty$ for some $\theta>0$, then, under the same conditions as in Theorem 1(i), i.e. $\bar{F}(x)^{-1} \sim \Phi\left(\bar{G}(x)^{-1}\right)$ with $\Phi(x)$ being regularly varying of index $\alpha>0$, we obtain, as $t \rightarrow \infty$,

$$
\mathbb{P}[T>t] \sim \frac{\Gamma(\alpha+1)(\mathbb{E}[U+A])^{\alpha}}{\Phi(t)} .
$$


Remark 14. $\mathbb{E}\left[L^{\alpha+\theta}\right]<\infty$ is close to a minimum condition for $\alpha>1$ since $\mathbb{E}\left[L^{\alpha-\theta}\right]=\infty$ implies that $\mathbb{E}\left[T^{\alpha-\theta}\right]=\infty$ in view of $T \geq L$, which would contradict (41).

The proof of Theorem 5 is presented in Subsection 3.5.

Theorem 6. Under the same conditions as in Theorem 2, i.e. $\Phi(x)=\mathrm{e}^{l(x)}$ satisfies (3), where $l(x)$ is slowly varying with

$$
\lim _{x \rightarrow \infty} \frac{l(x / l(x))}{l(x)}=1,
$$

and, in addition, if $\mathbb{E}[A]<\infty, \mathbb{P}[L>x]=O\left(\Phi(x)^{-(\delta+1)}\right), \mathbb{E}[U]<\infty$, and $x \mathbb{P}[U>x]=$ $O\left(\Phi(x)^{-(\delta+1)}\right)$ for some $\delta>0$, then we obtain

$$
\lim _{t \rightarrow \infty} \frac{\log \left(\mathbb{P}[T>t]^{-1}\right)}{\log (\Phi(t))}=1 .
$$

Remark 15. Theorem 6 implies parts (1:1), (2:1), and (2:2) of Theorem 2.1 of [1] and extends Theorem 2 of [8]. Furthermore, it shows that, if $\log \mathbb{P}[L>x]^{-1} \approx \alpha \log \mathbb{P}[A>x]^{-1}$, meaning that the hazard functions of $L$ and $A$ are asymptotically linear, the distribution tails of the number of transmissions and total transmission time are essentially power laws. Thus, the system can exhibit high variations and possible instability, e.g. when $0<\alpha<2$, the transmission time has an infinite variance and, when $0<\alpha<1$, it does not even have a finite mean.

Remark 16. It is easy to understand that, if the data sizes (e.g. files, packets) follow heavytailed distributions, the total transmission time is also heavy tailed. However, from Theorems 5 and 6, we see that even if the distributions of the channel characteristics and data are highly concentrated, e.g. when they are asymptotically proportional on the logarithmic scale (see Corollary 2 in Subsection 2.1.1), the heavy-tailed transmission delays can still arise.

The proof of Theorem 6 is presented in Subsection 3.6.

Theorem 7. Under the same conditions as in Theorem 3, i.e. $\Phi(x)=\mathrm{e}^{R_{\beta}(x)}$ satisfies (3), where $R_{\beta}(x)=x^{\beta} l(x), \beta>0$, is regularly varying with $l(x)$ satisfying

$$
\lim _{x \rightarrow \infty} \frac{l\left((x / l(x))^{1 /(1+\beta)}\right)}{l(x)}=1,
$$

and, in addition, if $\mathbb{E}[A]<\infty, \mathbb{P}[U>x]=O\left(\exp \left(-(\log \Phi(x))^{(1+\delta) /(\beta+1)}\right)\right)$ for $\delta>0$, $\mathbb{P}[L>x]=O\left(\mathrm{e}^{-x^{\xi}}\right)$, and $\mathbb{P}[A>x]=O\left(\mathrm{e}^{-x^{\zeta}}\right)$, where $\zeta \geq 0, \xi>\beta /(\beta+1)$, and $(1-\zeta) \beta<\xi$, then we obtain

$$
\lim _{t \rightarrow \infty} \frac{\log \left(\mathbb{P}[T>t]^{-1}\right)}{(\log \Phi(t))^{1 /(\beta+1)}}=\frac{\beta^{1 /(\beta+1)}+\beta^{-\beta /(\beta+1)}}{(\mathbb{E}[A+U])^{\beta /(\beta+1)}} .
$$

Remark 17. Theorem 7 implies part (1:2) of Theorem 2.1 of [1], and provides a more precise logarithmic asymptotic instead of a double logarithmic limit. Furthermore, it is easy to check that the condition $(1-\zeta) \beta<\xi$ holds in two special cases: (i) if $\zeta \geq \beta /(\beta+1)$ and $\xi>\beta /(\beta+1)$, and (ii) if $\xi>\beta$ and $\zeta=0$ (assuming no conditions for $\mathbb{P}[A>x]$ beyond $\mathbb{E}[A]<\infty)$.

The proof of Theorem 7 is presented in Subsection 3.7. Basically, the condition $(1-\zeta) \beta<\xi$ is needed since the following proposition shows that $\mathbb{P}[T>t]$ could have a heavier tail than predicted by (43) if $(1-\zeta) \beta>\xi$ (or, equivalently, $\xi /(\xi+1-\zeta)<\beta /(\beta+1)$ when $\zeta<1$ ). 
Proposition 8. If $\mathbb{P}[L>x]=\mathrm{e}^{-x^{\xi}}$ and $\mathbb{P}[A>x]=\mathrm{e}^{-x^{\zeta}}$ with $0<\xi, \zeta<1$, then, as $t \rightarrow \infty$,

$$
\mathbb{P}[T>t] \gtrsim \mathrm{e}^{-2 t^{\xi /(\xi+1-\zeta)}} .
$$

Proof. It is easy to see that, for $\delta, y>0$,

$$
\begin{aligned}
\mathbb{P}[T>t] & \geq \mathbb{P}\left[T>t, y<A_{i}<(1+\delta) y, 1 \leq i \leq \frac{t}{y}, L>(1+\delta) y\right] \\
& \geq(\mathbb{P}[y<A<(1+\delta) y])^{t / y} \mathbb{P}[L>(1+\delta) y],
\end{aligned}
$$

which, by noting that $\mathbb{P}[A>x]=\mathrm{e}^{-x^{\zeta}}$ with $\zeta>0$, yields

$$
\mathbb{P}[T>t] \gtrsim(\mathbb{P}[A>y])^{t / y} \mathbb{P}[L>(1+\delta) y]=\mathrm{e}^{-\left(t y^{\zeta} / y+y^{\xi}\right)} .
$$

Choosing $y=t^{1 /(\xi+1-\zeta)}$ completes the proof.

If $(1-\zeta) \beta>\xi$ then the exponent on the right-hand side of (44) satisfies $\xi /(\xi+1-\zeta)<$ $\beta /(\beta+1)$, which would contradict (43).

\section{Proofs}

\subsection{Proof of Proposition 2}

If $\log (\Phi(x))$ is slowly varying then, for any $0<\delta<\varepsilon<1$, there exists $x_{\delta}>0$ such that $\log (\Phi(x))<x^{\delta}$ for all $x>x_{\delta}$. By using condition (3), or, equivalently, (20), we obtain, for large enough $n$,

$$
\begin{aligned}
\mathbb{P}[N>n] & =\mathbb{E}\left[(1-\bar{G}(L))^{n}\right] \\
& \geq\left(1-\frac{1}{n}\right)^{n} \mathbb{P}\left[\bar{G}(L) \leq \frac{1}{n}\right] \\
& \geq\left(1-\frac{1}{n}\right)^{n} \mathbb{P}\left[\Phi^{\leftarrow}\left(\bar{F}^{-(1-\varepsilon)}(L)\right) \geq n\right] \\
& \geq\left(1-\frac{1}{n}\right)^{n} \mathrm{e}^{-n^{\delta} /(1-\varepsilon)},
\end{aligned}
$$

where we have used the fact that, for $x_{\varepsilon}$ chosen as in (20), we can always select $n$ large enough such that $\{\bar{G}(L) \leq 1 / n\} \subset\left\{L>x_{\varepsilon}\right\}$. Therefore, we obtain

$$
0 \leq \varlimsup_{n \rightarrow \infty} \frac{-\log \mathbb{P}[N>n]}{n^{\varepsilon}} \leq \lim _{n \rightarrow \infty} \frac{1+n^{\delta} /(1-\varepsilon)}{n^{\varepsilon}}=0,
$$

which proves the proposition.

\subsection{Continuation of the proof of Theorem 1}

Now, we prove the lower bound. For $K>0,1>\varepsilon>0$, and $x_{\varepsilon}$ selected as in (9), choosing $x_{n}>x_{\varepsilon}$ with $\Phi^{\leftarrow}\left((1-\varepsilon) \bar{F}\left(x_{n}\right)\right)=n / K$, we obtain, for large $n$,

$$
\begin{aligned}
\mathbb{P}[N>n] & =\mathbb{E}\left[(1-\bar{G}(L))^{n}\right] \\
& \geq \mathbb{E}\left[(1-\bar{G}(L))^{n} \mathbf{1}\left(L>x_{n}\right)\right] \\
& \geq \mathbb{E}\left[\left(1-\frac{1}{\Phi^{\leftarrow}\left((1-\varepsilon) V^{-1}\right)}\right)^{n} \mathbf{1}\left(V<\bar{F}\left(x_{n}\right)\right)\right],
\end{aligned}
$$


where $V$ is a uniform random variable on $(0,1)$. Recall that, without loss of generality, $\Phi(\cdot)$ can be assumed to be absolutely continuous and differentiable. Thus, by letting $z=$ $n / \Phi^{\leftarrow}\left((1-\varepsilon) u^{-1}\right)$, we obtain

$$
\begin{aligned}
\mathbb{P}[N>n] \Phi(n) & \geq \Phi(n) \int_{0}^{\bar{F}\left(x_{n}\right)}\left(1-\frac{1}{\Phi^{\leftarrow}((1-\varepsilon) / u)}\right)^{n} \mathrm{~d} u \\
& \geq \int_{\varepsilon}^{K}\left(1-\frac{z}{n}\right)^{n} \frac{\Phi(n)}{\Phi(n / z)} \frac{\Phi^{\prime}(n / z)}{\Phi(n / z)} \frac{(1-\varepsilon) n}{z^{2}} \mathrm{~d} z .
\end{aligned}
$$

From (45), following the same approach as used to derive (16), we obtain, as $n \rightarrow \infty$,

$$
\mathbb{P}[N>n] \Phi(n) \sim \int_{\varepsilon}^{K}(1-\varepsilon) \alpha \mathrm{e}^{-z} z^{\alpha-1} \mathrm{~d} z
$$

which, passing $K \rightarrow \infty$ and $\varepsilon \rightarrow 0$, yields

$$
\mathbb{P}[N>n] \Phi(n) \gtrsim \int_{0}^{\infty} \alpha \mathrm{e}^{-z} z^{\alpha-1} \mathrm{~d} z=\Gamma(\alpha+1) .
$$

Combining (17) and (46) completes the proof of (5).

We now proceed to the proof of (6). We begin by proving the lower bound. Recalling (9), for large enough $n$ and $\varepsilon>0$, we obtain

$$
\begin{aligned}
\mathbb{P}[N>n] & =\mathbb{E}\left[(1-\bar{G}(L))^{n}\right] \\
& \geq\left(1-\frac{\varepsilon}{n}\right)^{n} \mathbb{P}\left[\bar{G}(L) \leq \frac{\varepsilon}{n}\right] \\
& \geq\left(1-\frac{\varepsilon}{n}\right)^{n} \mathbb{P}\left[\Phi \leftarrow\left((1-\varepsilon) \bar{F}^{-1}(L)\right) \geq \frac{n}{\varepsilon}\right] \\
& \geq\left(1-\frac{\varepsilon}{n}\right)^{n} \frac{1-\varepsilon}{\Phi(n / \varepsilon)},
\end{aligned}
$$

implying that

$$
\varliminf_{n \rightarrow \infty} \mathbb{P}[N>n] \Phi(n) \geq \lim _{n \rightarrow \infty}\left(1-\frac{\varepsilon}{n}\right)^{n} \frac{(1-\varepsilon) \Phi(n)}{\Phi(n / \varepsilon)}=(1-\varepsilon) \mathrm{e}^{-\varepsilon},
$$

which, by passing $\varepsilon \rightarrow 0$, yields

$$
\varliminf_{n \rightarrow \infty} \mathbb{P}[N>n] \Phi(n) \geq 1 .
$$

Let us now prove the upper bound. Following an approach similar to that used to derive (10), we obtain

$$
\begin{aligned}
\mathbb{P}[N>n] \leq & \mathbb{E}\left[\mathrm{e}^{-n / \Phi^{\leftarrow}\left((1+\varepsilon) V^{-1}\right)}\right]+\left(1-\bar{G}\left(x_{\varepsilon}\right)\right)^{n} \\
\leq & \mathbb{P}\left[0 \leq \frac{n}{\Phi^{\leftarrow}\left((1+\varepsilon) V^{-1}\right)} \leq \mathrm{e}^{m}\right] \\
& +\sum_{k=m}^{\lceil\log (\varepsilon n)\rceil} \mathrm{e}^{-\mathrm{e}^{k}} \mathbb{P}\left[\mathrm{e}^{k} \leq \frac{n}{\Phi^{\leftarrow}\left((1+\varepsilon) V^{-1}\right)} \leq \mathrm{e}^{k+1}\right]+o\left(\frac{1}{\Phi(n)}\right) \\
\leq & \frac{1+\varepsilon}{\Phi\left(n / \mathrm{e}^{m}\right)}+\sum_{k=m}^{\lceil\log (\varepsilon n)\rceil} \mathrm{e}^{-\mathrm{e}^{k}} \frac{1+\varepsilon}{\Phi\left(n / \mathrm{e}^{k+1}\right)}+o\left(\frac{1}{\Phi(n)}\right),
\end{aligned}
$$


resulting in

$$
\mathbb{P}[N>n] \Phi(n) \leq \frac{(1+\varepsilon) \Phi(n)}{\Phi\left(n / \mathrm{e}^{m}\right)}+\sum_{k=m}^{\lceil\log (\varepsilon n)\rceil} \mathrm{e}^{-\mathrm{e}^{k}} \frac{(1+\varepsilon) \Phi(n)}{\Phi\left(n / \mathrm{e}^{k+1}\right)}+o(1) .
$$

Note that the second term on the right-hand side of (48) is always finite because of (11) and, by passing $n \rightarrow \infty$ and then $m \rightarrow \infty$ in (48), we obtain

$$
\varlimsup_{n \rightarrow \infty} \mathbb{P}[N>n] \Phi(n) \leq 1 \text {. }
$$

Combining (47) and (49) completes the proof of (6).

\subsection{Proof of Proposition 5}

We first prove the lower bound. By recalling condition (4), or, equivalently, (9), and using $1-x \geq \mathrm{e}^{-(1+\varepsilon) x}$ for small enough $x$, we obtain, for large enough $n$ and $x_{n}=o(n)>0$,

$$
\begin{aligned}
\mathbb{P}[N>n] & \geq \mathbb{E}\left[(1-\bar{G}(L))^{n} \mathbf{1}\left(L \geq x_{\varepsilon}\right)\right] \\
& \geq \mathbb{E}\left[\mathrm{e}^{-(1+\varepsilon) \bar{G}(L) n} \mathbf{1}\left(L \geq x_{\varepsilon}\right)\right] \\
& \geq \mathbb{E}\left[\mathrm{e}^{-(1+\varepsilon) n / \Phi \leftarrow\left((1-\varepsilon) V^{-1}\right)} \mathbf{1}\left(V \leq \bar{F}\left(x_{\varepsilon}\right)\right)\right] \\
& \geq \mathrm{e}^{-x_{n}} \mathbb{P}\left[\frac{(1+\varepsilon) n}{\Phi^{\leftarrow}\left((1-\varepsilon) V^{-1}\right)} \leq x_{n}, V \leq \bar{F}\left(x_{\varepsilon}\right)\right] \\
& =\mathrm{e}^{-x_{n}}(1-\varepsilon) \Phi\left(\frac{(1+\varepsilon) n}{x_{n}}\right)^{-1} \\
& =(1-\varepsilon) \mathrm{e}^{-x_{n}-\lambda\left(\log n-\log \left(x_{n} /(1+\varepsilon)\right)\right)^{\delta}} .
\end{aligned}
$$

Using the preceding inequality and setting $x_{n}=\lambda \delta(\log n)^{\delta-1}$ yields, for large enough $n$,

$$
\begin{aligned}
\log \mathbb{P}[N>n]^{-1}-\lambda(\log n)^{\delta} & \leq \lambda\left(\log n-\log \left(\frac{x_{n}}{1+\varepsilon}\right)\right)^{\delta}-\lambda(\log n)^{\delta}+x_{n}-\log (1-\varepsilon) \\
& \leq-(1-\varepsilon) \lambda \delta(\log n)^{\delta-1} \log \left(\lambda \delta(\log n)^{\delta-1}\right)+\lambda \delta(\log n)^{\delta-1}
\end{aligned}
$$

which, by passing $n \rightarrow \infty$ and then $\varepsilon \rightarrow 0$, results in

$$
\log \mathbb{P}[N>n]^{-1}-\lambda(\log n)^{\delta} \lesssim-\lambda \delta(\delta-1)(\log \log n)(\log n)^{\delta-1} .
$$

Let us now prove the upper bound. Following the same approach as used in the proof of Theorem 2, we obtain, for large $n$ and $y_{n}=\lambda(\log n)^{\delta}-\lambda \delta(\delta-1) \log \log n(\log n)^{\delta-1}$,

$$
\begin{aligned}
\mathbb{P}[N>n] & \leq \sum_{k=0}^{y_{n}-1} \mathrm{e}^{-k} \mathbb{P}\left[k \leq \frac{n}{\Phi^{\leftarrow}\left((1+\varepsilon) V^{-1}\right)} \leq k+1\right]+\mathrm{e}^{-y_{n}}+o(\mathbb{P}[N>n]) \\
& \leq(1+\varepsilon) \sum_{k=0}^{y_{n}-1} \mathrm{e}^{-k-\lambda(\log n-\log (k+1))^{\delta}}+\mathrm{e}^{-y_{n}}+o(\mathbb{P}[N>n]) .
\end{aligned}
$$

For $f(x)=x+\lambda(\log n-\log x)^{\delta}$, it is easy to check that $f^{\prime}(x)=1-\lambda \delta(\log n-\log x)^{\delta-1} / x$ is monotonically increasing in $x$ on $(0, n)$. Then, by defining

$$
x_{1}:=\lambda \delta(\log n)^{\delta-1}-(1-\varepsilon) \lambda \delta(\delta-1)^{2}(\log \log n)(\log n)^{\delta-2}, \quad \varepsilon>0,
$$


we obtain, after some calculations, for large $n$,

$$
f^{\prime}\left(x_{1}\right) \geq 1-\frac{(\log n)^{\delta-1}-(1-\varepsilon / 2)(\delta-1)^{2}(\log \log n)(\log n)^{\delta-2}}{(\log n)^{\delta-1}-(1-\varepsilon)(\delta-1)^{2}(\log \log n)(\log n)^{\delta-2}}>0,
$$

which implies that $f^{\prime}(x)>0$ for $x \geq x_{1}$ and, therefore, inf $x_{x \in\left[x_{1}, n\right)} \geq f\left(x_{1}\right)$. In addition, $f\left(x_{1}\right) \geq \inf _{0<x \leq x_{1}} f(x) \geq \lambda\left(\log n-\log x_{1}\right)^{\delta}$. Hence, by (51) we obtain

$$
\mathbb{P}[N>n] \leq(1+\varepsilon) y_{n} \mathrm{e}^{1-\lambda\left(\log n-\log x_{1}\right)^{\delta}}+\mathrm{e}^{-y_{n}}+o(\mathbb{P}[N>n]),
$$

which, by recalling the definitions of $y_{n}$ and $x_{1}$, results in

$$
\log \mathbb{P}[N>n]^{-1}-\lambda(\log n)^{\delta} \gtrsim-(1+\varepsilon) \lambda \delta(\delta-1)(\log \log n)(\log n)^{\delta-1} .
$$

Finally, passing $\varepsilon \rightarrow 0$ in (52) and combining it with (50), we complete the proof.

\subsection{Proof of Proposition 6}

We first prove the lower bound. Applying the same arguments as used in the proof of the lower bound for Theorem 3, we obtain, for $0<\varepsilon<1, x_{n}>0$, and large enough $n$,

$$
\log \left(\mathbb{P}[N>n]^{-1}\right) \leq x_{n}+\frac{1}{1-\varepsilon} \log \left(\Phi\left(\frac{(1+\varepsilon) n}{x_{n}}\right)\right)=x_{n}+\frac{1}{1-\varepsilon} \mathrm{e}^{\lambda\left(\log \left((1+\varepsilon) n / x_{n}\right)\right)^{\delta}} .
$$

Setting $x_{n}=\mathrm{e}^{\lambda(\log n)^{\delta}\left(1-\delta \lambda(\log n)^{\delta-1}\right)}, \frac{1}{2}<\delta<1$, in the preceding inequality yields

$$
\log \left(\mathbb{P}[N>n]^{-1}\right) \leq \mathrm{e}^{\lambda(\log n)^{\delta}\left(1-\delta \lambda(\log n)^{\delta-1}\right)}+\frac{1}{1-\varepsilon} \mathrm{e}^{\lambda\left(\log n-\log x_{n}+\log (1+\varepsilon)\right)^{\delta}},
$$

which, by noting that $\lambda\left(\log n-\log x_{n}+\log (1+\varepsilon)\right)^{\delta} \leq \lambda(\log n)^{\delta}\left(1-(1-\varepsilon) \delta \lambda(\log n)^{\delta-1}\right)$ for all large enough $n$, implies that, for large enough $n$,

$$
\log \left(\log \mathbb{P}[N>n]^{-1}\right) \leq \log \left(1+\frac{1}{1-\varepsilon}\right)+\lambda(\log n)^{\delta}\left(1-(1-\varepsilon) \delta \lambda(\log n)^{\delta-1}\right) .
$$

Passing $\varepsilon \rightarrow 0$ in the preceding inequality results in

$$
\log \left(\log \mathbb{P}[N>n]^{-1}\right)-\lambda(\log n)^{\delta} \lesssim-\delta \lambda^{2}(\log n)^{2 \delta-1} .
$$

Let us now prove the upper bound. Following the same approach as used in the proof of Theorem 2, we obtain

$$
\mathbb{P}[N>n] \leq \sum_{k=0}^{y_{n}-1} \exp \left(-k-\frac{1}{1+\varepsilon} \mathrm{e}^{\lambda(\log n-\log k)^{\delta}}\right)+\mathrm{e}^{-y_{n}}+o(\mathbb{P}[N>n]) .
$$

Choose $y_{n}=\mathrm{e}^{\lambda(\log n)^{\delta}\left(1-(1+\varepsilon) \delta \lambda(\log n)^{\delta-1}\right)}$, and let $f(x)=x+\mathrm{e}^{\lambda(\log n-\log x)^{\delta}} /(1+\varepsilon)$. Since $f^{\prime}(x)$ is an increasing function in $x$ on $(0, n)$, it is can be shown that $f^{\prime}(x) \leq f^{\prime}\left(y_{n}\right)<0$ for all $0<x \leq y_{n}$ and large enough $n$. Therefore, for $0 \leq k \leq y_{n}$, we obtain

$$
\exp \left(-k-\frac{1}{1+\varepsilon} \mathrm{e}^{\lambda(\log n-\log k)^{\delta}}\right) \leq \exp \left(-y_{n}-\frac{1}{1+\varepsilon} \mathrm{e}^{\lambda\left(\log n-\log y_{n}\right)^{\delta}}\right),
$$


which, by (54), yields

$$
\begin{aligned}
\mathbb{P}[N>n] & \leq y_{n} \exp \left(-y_{n}-\frac{1}{1+\varepsilon} \mathrm{e}^{\lambda\left(\log n-\log y_{n}\right)^{\delta}}\right)+\mathrm{e}^{-y_{n}}+o(\mathbb{P}[N>n]) \\
& \leq\left(y_{n}+1\right) \mathrm{e}^{-y_{n}}+o(\mathbb{P}[N>n]),
\end{aligned}
$$

implying that

$$
\log \left(\log \mathbb{P}[N>n]^{-1}\right)-\lambda(\log n)^{\delta} \gtrsim-(1+\varepsilon) \delta \lambda^{2}(\log n)^{2 \delta-1} .
$$

Finally, by passing $\varepsilon \rightarrow 0$ in (55) and combining it with (53), we complete the proof.

\subsection{Proof of Theorem 5}

The proof is based on large deviation results developed by Nagaev in [11]; specifically, we summarize Corollary 1.6 and Corollary 1.8 of [11] in the following lemma.

Lemma 1. Let $X_{1}, X_{2}, \ldots, X_{n}$, and $X$ be independent and identically distributed random variables with $\int_{u \geq 0} u^{s} \mathrm{~d} \mathbb{P}[X<u]<\infty$ and $\mathbb{E} X=0$.

- If $1 \leq s \leq 2$ then there exist finite $y_{s}, c>0$ such that, for $x>y>y_{s}$,

$$
\mathbb{P}\left[\sum_{i=1}^{n} X_{i} \geq x\right] \leq n \mathbb{P}[X>y]+\left(\frac{c n}{x y^{s-1}}\right)^{x / 2 y} .
$$

- If $s>2$ then there exists finite $c>0$ such that

$$
\mathbb{P}\left[\sum_{i=1}^{n} X_{i} \geq x\right] \leq \frac{c n}{x^{s}}+\exp \left(\frac{-x^{2}}{c n}\right) .
$$

We are now ready to prove Theorem 5.

Proof of Theorem 5. We first establish the upper bound. By recalling Definition 1, for any $\frac{1}{2}>\delta>0$, we obtain

$$
\begin{aligned}
\mathbb{P}[T>t]= & \mathbb{P}\left[\sum_{i=1}^{N-1}\left(U_{i}+A_{i}\right)+L>t\right] \\
\leq & \mathbb{P}\left[\sum_{i=1}^{N}\left(A_{i} \wedge L+\mathbb{E}[U]\right)>(1-2 \delta) t\right]+\mathbb{P}\left[\sum_{i=1}^{N}\left(U_{i}-\mathbb{E}[U]\right)>\delta t\right] \\
& +\mathbb{P}[L>\delta t] \\
= & : I_{1}+I_{2}+I_{3} .
\end{aligned}
$$

The condition $\mathbb{E}\left[L^{\alpha+\theta}\right]<\infty$ implies that

$$
I_{3} \leq \frac{\mathbb{E}\left[L^{\alpha+\theta}\right]}{(\delta t)^{\alpha+\theta}}=O\left(\frac{1}{t^{\alpha+\theta}}\right) .
$$

Let us consider $I_{2}$. Suppose that $\alpha>1$, i.e. when $\mathbb{E}[N]<\infty$. Since $N$ is independent of $\left\{U_{i}\right\}$, by defining $X_{i}:=U_{i}-\mathbb{E}\left[U_{i}\right]$, we obtain

$$
I_{2}=\sum_{n=1}^{\infty} \mathbb{P}[N=n] \mathbb{P}\left[\sum_{i=1}^{n} X_{i}>\delta t\right]
$$


To evaluate $\mathbb{P}\left[\sum_{i=1}^{n} X_{i}>\delta t\right]$ in the preceding equality, we need to apply Lemma 1 , which results in two situations. If $1<s:=\alpha+\theta \leq 2$, using (56) with $y=\delta t / 2$, we obtain, for all $n \geq 1$,

$$
\mathbb{P}\left[\sum_{i=1}^{n} X_{i}>\delta t\right] \leq n \mathbb{P}\left[X_{1}>\frac{\delta t}{2}\right]+\frac{2^{s-1} c n}{\delta^{s} t^{s}},
$$

implying that

$$
\begin{aligned}
I_{2} & \leq \sum_{n=1}^{\infty} \mathbb{P}[N=n]\left(n \mathbb{P}\left[X_{1}>\frac{\delta t}{2}\right]+\frac{2^{s-1} c n}{\delta^{s} t^{s}}\right) \\
& \leq \mathbb{E}[N] \mathbb{P}\left[X_{1}>\frac{\delta t}{2}\right]+\frac{2^{s-1} c \mathbb{E}[N]}{\delta^{s} t^{\alpha+\theta}} \\
& =O\left(\frac{1}{t^{\alpha+\theta}}\right) .
\end{aligned}
$$

Otherwise, if $s=\alpha+\theta>2$, by (57), we obtain, for $0<\gamma<\alpha \delta /(1+\delta)$,

$$
\begin{aligned}
I_{2} & \leq \mathbb{P}\left[\sum_{i=1}^{\left\lfloor t^{1+\delta}\right\rfloor} X_{i}>\delta t\right]+\mathbb{P}\left[N>t^{1+\delta}\right] \\
& =\sum_{n=1}^{\left\lfloor t^{1+\delta}\right\rfloor} \mathbb{P}[N=n] \mathbb{P}\left[\sum_{i=1}^{n} X_{i}>\delta t\right]+O\left(\frac{1}{t^{(1+\delta)(\alpha-\gamma)}}\right) \\
& \leq \frac{c \mathbb{E}[N]}{(\delta t)^{\alpha+\theta}}+\exp \left(-\frac{\delta^{2} t^{1-\delta}}{c}\right)+O\left(\frac{1}{t^{(1+\delta)(\alpha-\gamma)}}\right),
\end{aligned}
$$

which implies that, for some $v>0$,

$$
I_{2}=O\left(\frac{1}{t^{\alpha+v}}\right)
$$

We now suppose that $0<\alpha \leq 1$. For $1<s:=1+\theta \leq 2, \theta>0$, recalling (60) and noting that $\sum_{n=1}^{\left\lfloor t^{\zeta}\right\rfloor} n \mathbb{P}[N=n] \leq H t^{\zeta(1-\alpha+\sigma)}$ for $\alpha>\theta>\sigma>0,(\theta+1) /(\sigma+1)>\zeta>1$, and large enough $H$, we obtain, for some $v>0$,

$$
\begin{aligned}
I_{2} & \leq \sum_{n=1}^{\left\lfloor t^{\zeta}\right\rfloor} \mathbb{P}[N=n]\left(n \mathbb{P}\left[X_{1}>\frac{\delta t}{2}\right]+\frac{2^{s-1} c n}{\delta^{s} t^{s}}\right)+\mathbb{P}\left[N>t^{\zeta}\right] \\
& \leq H t^{\zeta(1-\alpha+\sigma)}\left(\frac{\mathbb{E}\left[X_{1}^{1+\theta}\right]}{(\delta t / 2)^{1+\theta}}+\frac{2^{s-1} c}{\delta^{s} t^{1+\theta}}\right)+\mathbb{P}\left[N>t^{\zeta}\right] \\
& =O\left(\frac{1}{t^{\alpha+v}}\right),
\end{aligned}
$$

which, together with (61) and (62), yields, for some $v>0$,

$$
I_{2}=O\left(\frac{1}{t^{\alpha+v}}\right)
$$


Let us now consider $I_{1}$. It is easy to obtain, for $\varepsilon>0$,

$$
\begin{aligned}
I_{1} \leq & \mathbb{P}\left[\sum_{i=1}^{(1-2 \delta) t / \mathbb{E}[A+U](1+\delta)}\left(A_{i} \wedge(\varepsilon t)+\mathbb{E}[U]\right)>(1-2 \delta) t\right] \\
& +\mathbb{P}\left[N>\frac{(1-2 \delta) t}{\mathbb{E}[A+U](1+\delta)}\right]+\mathbb{P}[L>\varepsilon t] \\
= & : I_{11}+I_{12}+I_{13} .
\end{aligned}
$$

By recalling Theorem 1, we know that

$$
\mathbb{P}\left[N>\frac{(1-3 \delta) t}{\mathbb{E}[A+U](1+\delta)}\right] \sim \frac{\Gamma(\alpha+1)(\mathbb{E}[U+A](1+\delta))^{\alpha}}{\Phi(t)(1-3 \delta)^{\alpha}} .
$$

The same argument as that used in (59) implies that

$$
I_{13}=O\left(\frac{1}{t^{\alpha+\theta}}\right) \text {. }
$$

Furthermore, $I_{11}$ is upper bounded by

$$
\begin{aligned}
& \mathbb{P}\left[\sum_{i=1}^{(1-3 \delta) t / \mathbb{E}[A+U](1+\delta)}\left(A_{i} \wedge(\varepsilon t)+\mathbb{E}[U]\right)-(1+\delta) \mathbb{E}[A+U] \frac{(1-3 \delta) t}{\mathbb{E}[A+U](1+\delta)}>\delta t\right] \\
& \leq \mathbb{P}\left[\sup _{n}\left\{\sum_{i=1}^{n}\left(A_{i} \wedge(\varepsilon t)+\mathbb{E}[U]\right)-n(1+\delta) \mathbb{E}[A+U]\right\}>\delta t\right],
\end{aligned}
$$

where in the above probability, $\sup _{n}\left\{\sum_{i=1}^{n}\left(A_{i} \wedge(\varepsilon t)+\mathbb{E}[U]\right)-n(1+\delta) \mathbb{E}[A+U]\right\}$ is equal in distribution to the stationary workload in a $\mathrm{D} / \mathrm{GI} / 1$ queue with truncated service times with the stability condition $\mathbb{E}[(A \wedge(\varepsilon t)+\mathbb{E}[U])]<(1+\delta) \mathbb{E}[A+U]$. Therefore, applying similar arguments to those used in the proof of Lemma 3.2 of [5], for any fixed $\beta>0$, we can choose $\varepsilon>0$ small enough in (67) such that, as $t \rightarrow \infty$,

$$
I_{11}=o\left(\frac{1}{t^{\beta}}\right)
$$

which, together with (58), (59), (63), (64), (65), and (66), yields, by passing $\varepsilon, \delta \rightarrow 0$ in (65),

$$
\mathbb{P}[T>t] \lesssim \frac{\Gamma(\alpha+1)(\mathbb{E}[U+A])^{\alpha}}{\Phi(t)} .
$$

We now prove the lower bound. It is easy to obtain, for $\delta>0$,

$$
\begin{aligned}
\mathbb{P}[T>t] & =\mathbb{P}\left[\sum_{i=1}^{N-1}\left(U_{i}+A_{i}\right)+L>t\right] \\
& \geq \mathbb{P}\left[\sum_{i=1}^{N-1}\left(U_{i}+A_{i}\right)>t, N \geq \frac{t(1+\delta)}{\mathbb{E}[U+A]}+1\right] \\
& \geq \mathbb{P}\left[N \geq \frac{t(1+\delta)}{\mathbb{E}[U+A]}+1\right]-\mathbb{P}\left[\sum_{i=1}^{N-1}\left(U_{i}+A_{i}\right) \leq t, N \geq \frac{t(1+\delta)}{\mathbb{E}[U+A]}+1\right] \\
& =: I_{1}-I_{2} .
\end{aligned}
$$


For $I_{2}$, by defining $Y_{i}:=\mathbb{E}[U+A]-U_{i}-A_{i}$, we obtain

$$
I_{2} \leq \mathbb{P}\left[\sum_{i \leq t(1+\delta) / \mathbb{E}[U+A]}\left(U_{i}+A_{i}\right) \leq t\right]=\mathbb{P}\left[\sum_{i \leq t(1+\delta) / \mathbb{E}[U+A]} Y_{i} \geq \delta t\right]
$$

with $Y_{i} \leq \mathbb{E}[U+A]<\infty$. By Chernoff's bound, there exist $h, \eta>0$ such that

$$
I_{2} \leq O\left(h \mathrm{e}^{-\eta t}\right)
$$

which, by Theorem 1, (69), and passing $\delta \rightarrow 0$, yields

$$
\mathbb{P}[T>t] \gtrsim \frac{\Gamma(\alpha+1)(\mathbb{E}[U+A])^{\alpha}}{\Phi(t)} .
$$

Combining (68) and (71) completes the proof.

\subsection{Proof of Theorem 6}

We first prove the upper bound. It is easy to see that

$$
\begin{aligned}
\mathbb{P}[T>t]= & \mathbb{P}\left[\sum_{i=1}^{N-1}\left(\left(A_{i} \wedge L\right)+U_{i}\right)+L>t\right] \\
\leq & \mathbb{P}\left[\sum_{i=1}^{\lceil t / l(t)\rceil}\left(A_{i} \wedge L\right)>\frac{t}{3}\right]+\mathbb{P}\left[\sum_{i=1}^{\lceil t / l(t)\rceil} U_{i}>\frac{t}{3}\right]+\mathbb{P}\left[N>\left\lceil\frac{t}{l(t)}\right]+1\right] \\
& +\mathbb{P}\left[L>\frac{t}{3}\right] \\
= & : I_{1}+I_{2}+I_{3}+I_{4} .
\end{aligned}
$$

Now, since $l(\cdot)$ is slowly varying and $\mathbb{P}[L>x]=O\left(\Phi(x)^{-(\delta+1)}\right)$, we obtain

$$
I_{4}=\mathbb{P}\left[L>\frac{t}{3}\right]=o\left(\Phi(t)^{-1}\right)
$$

By Theorem 2 we obtain

$$
\lim _{t \rightarrow \infty} \frac{\log \left(\mathbb{P}[N>\lceil t / l(t)\rceil+1]^{-1}\right)}{\log \Phi(t / l(t))}=1
$$

which, by (42), yields

$$
\lim _{t \rightarrow \infty} \frac{\log \left(\mathbb{P}[N>\lceil t / l(t)\rceil+1]^{-1}\right)}{\log (\Phi(t))}=1 .
$$

To evaluate $I_{2}$, we will use the decomposition

$$
\begin{aligned}
I_{2} & =\mathbb{P}\left[\sum_{i=1}^{\lceil t / l(t)\rceil} U_{i}>\frac{t}{3}\right] \\
& \leq\left\lceil\frac{t}{l(t)}\right] \mathbb{P}\left[U_{1}>\frac{t}{l(t)}\right]+\mathbb{P}\left[\sum_{i=1}^{\lceil t / l(t)\rceil} U_{i} \wedge \frac{t}{l(t)}>\frac{t}{3}\right] \\
& =: I_{21}+I_{22} .
\end{aligned}
$$


For $0<\delta<1$ and large $t$, owing to condition (42), we obtain $l(t / l(t)) \geq(1-\delta / 2) l(t)$, which yields

$$
I_{21} \leq O\left(\mathrm{e}^{-(1+\delta) l(t / l(t))}\right) \leq O\left(\mathrm{e}^{-(1+\delta)(1-\delta / 2) l(t)}\right)=o\left(\Phi(t)^{-1}\right) .
$$

Then, by using Chernoff's bound, for $h>0$, we obtain

$$
\begin{aligned}
I_{22} & =\mathbb{P}\left[\exp \left(h\left(\sum_{i=1}^{\lceil t / l(t)\rceil} U_{i} \wedge \frac{t}{l(t)}\right)\right)>\mathrm{e}^{h t / 3}\right] \\
& \leq \mathrm{e}^{-h t / 3}\left(\mathbb{E}\left[\mathrm{e}^{h\left(U_{i} \wedge t / l(t)\right)}\right]\right)^{t / l(t)+1},
\end{aligned}
$$

which, by selecting $h=6 l(t) / t$, using $\mathrm{e}^{x} \leq 1+x \mathrm{e}^{6} / 6$ for $0 \leq x \leq 6$, and noting that $\mathbb{E}[U]<\infty$, implies that

$$
\begin{aligned}
I_{22} & \leq \mathrm{e}^{-2 l(t)}\left(\mathbb{E}\left[1+\frac{\mathrm{e}^{6} l(t)}{t}\left(U_{1} \wedge \frac{t}{l(t)}\right)\right]\right)^{t / l(t)+1} \\
& \leq \mathrm{e}^{-2 l(t)}\left(1+\frac{\mathrm{e}^{6} l(t)}{t} \mathbb{E}\left[U_{1}\right]\right)^{t / l(t)+1} \\
& =o\left(\frac{1}{\Phi(t)}\right)
\end{aligned}
$$

Combining (75), (76), and (77) yields

$$
I_{2}=o\left(\frac{1}{\Phi(t)}\right)
$$

To evaluate $I_{1}$, we will use the decomposition

$$
\begin{aligned}
I_{1} & =\mathbb{P}\left[\sum_{i=1}^{\lceil t / l(t)\rceil}\left(A_{i} \wedge L\right)>\frac{t}{3}\right] \\
& \leq \mathbb{P}\left[\sum_{i=1}^{\lceil t / l(t)\rceil}\left(A_{i} \wedge \frac{t}{l(t)}\right)>\frac{t}{3}\right]+\mathbb{P}\left[L>\frac{t}{l(t)}\right] \\
& =: I_{11}+I_{12} .
\end{aligned}
$$

Applying the same argument as used in the derivation of (77) and the fact that $\mathbb{E}[A]<\infty$, we can prove that $I_{11}=o(1 / \Phi(t))$, which, by noting that condition (42) implies that $I_{12}=o(1 / \Phi(t))$, yields

$$
I_{1}=o\left(\frac{1}{\Phi(t)}\right) .
$$

Combining (72), (73), (74), (78), and (79) yields

$$
\varlimsup_{t \rightarrow \infty} \frac{\log \mathbb{P}[T>t]}{\log (\Phi(t))} \leq-1 .
$$


Let us now prove the lower bound. Observe that

$$
\begin{aligned}
\mathbb{P}\left[\sum_{i=1}^{N-1}\left(A_{i}+U_{i}\right)+L>t\right] & \geq \mathbb{P}\left[\sum_{i=0}^{N-1}\left(A_{i} \wedge 1\right)>t, N>\left\lceil\frac{2 t}{\mathbb{E}[A \wedge 1]}\right]+1\right] \\
& \geq \mathbb{P}\left[N>\left[\frac{2 t}{\mathbb{E}[A \wedge 1]}\right]+1\right]-\mathbb{P}\left[\sum_{i=1}^{\lceil 2 t / \mathbb{E}[A \wedge 1]\rceil}\left(A_{i} \wedge 1\right) \leq t\right]
\end{aligned}
$$

and, by applying the same arguments as used in the derivation of (70), it is easy to prove that the second probability on the right-hand side of the above inequality is exponentially bounded. Therefore, using Theorem 2 and the preceding exponential bound yields

$$
\varliminf_{t \rightarrow \infty} \frac{\log \mathbb{P}[T>t]}{\Phi(\log t)} \geq-1
$$

Combining (80) and (81) completes the proof.

\subsection{Proof of Theorem 7}

We first prove the upper bound. It is easy to see that, for $\eta:=\mathbb{E}[U] / \mathbb{E}[A+U]$ and $0<\varepsilon<1$,

$$
\begin{aligned}
\mathbb{P}[T>(1+\varepsilon) t]= & \mathbb{P}\left[\sum_{i=1}^{N-1}\left(\left(A_{i} \wedge L\right)+U_{i}\right)+L>(1+\varepsilon) t\right] \\
\leq & \mathbb{P}\left[\sum_{i=1}^{\lfloor(1-\varepsilon) t / \mathbb{E}[A+U]\rfloor}\left(A_{i} \wedge L\right)>(1-\eta) t\right]+\mathbb{P}\left[\sum_{i=1}^{\lfloor(1-\varepsilon) t / \mathbb{E}[A+U]\rfloor} U_{i}>\eta t\right] \\
& +\mathbb{P}\left[N>\left\lfloor\frac{(1-\varepsilon) t}{\mathbb{E}[A+U]}\right]\right]+\mathbb{P}[L>\varepsilon t] \\
= & : I_{1}+I_{2}+I_{3}+I_{4} .
\end{aligned}
$$

The condition on $L$ implies that

$$
I_{4}=\mathbb{P}[L>\varepsilon t]=o\left(\mathrm{e}^{-(\log \Phi(t))^{1 /(\beta+1)}}\right),
$$

and, by Theorem 3, we obtain

$$
\lim _{t \rightarrow \infty} \frac{\log \left(\mathbb{P}[N>\lfloor(1-\varepsilon) t / \mathbb{E}[A+U]\rfloor]^{-1}\right)}{(\log \Phi(t))^{1 /(\beta+1)}}=(1-\varepsilon)^{\beta /(\beta+1)} \frac{\beta^{1 /(\beta+1)}+\beta^{-\beta /(\beta+1)}}{(\mathbb{E}[A+U])^{\beta /(\beta+1)}} .
$$

Now, we evaluate $I_{2}$. By applying the large deviation result proved in Theorem 3.2(ii) of [5], and noting that $\mathbb{P}[U>x] \leq o\left(\mathrm{e}^{-x^{(1+\delta / 2) \beta /(\beta+1)}}\right)$, we can prove that there exist $1>\gamma>0$ and $C>0$ such that

$$
\begin{aligned}
\mathbb{P}\left[\sum_{i=1}^{\lfloor(1-\varepsilon) t / \mathbb{E}[A+U]\rfloor}\left(U_{i} \wedge \gamma \varepsilon \eta t\right)-\eta(1-\varepsilon) t>\varepsilon \eta t\right] & \leq C\left(\mathrm{e}^{-(\varepsilon \eta t)^{(1+\delta / 2) \beta /(\beta+1)}}\right) \\
& =o\left(\mathrm{e}^{-(\log \Phi(t))^{1 /(\beta+1)}}\right) .
\end{aligned}
$$


Thus, we obtain

$$
I_{2} \leq\left\lfloor\frac{(1-\varepsilon) t}{\mathbb{E}[A+U]}\right\rfloor \mathbb{P}\left[U_{1}>(\gamma \varepsilon \eta) t\right]+\mathbb{P}\left[\sum_{i=1}^{\lfloor(1-\varepsilon) t / \mathbb{E}[A+U]\rfloor}\left(U_{i} \wedge \gamma \varepsilon \eta t\right)>\eta t\right],
$$

which, by (85) and the assumption on $U$, yields

$$
I_{2}=o\left(\mathrm{e}^{-(\log \Phi(t))^{1 /(\beta+1)}}\right) .
$$

For $I_{1}$, we begin by proving the case in which $\zeta=0$ and $\xi>\beta$, i.e. assuming no conditions on $\mathbb{P}[A>x]$ beyond $\mathbb{E}[A]<\infty$. It is easy to obtain, for $0<\varepsilon<1 /(\beta+1)$,

$$
\begin{aligned}
I_{1} & =\mathbb{P}\left[\sum_{i=1}^{\lfloor(1-\varepsilon) t / \mathbb{E}[A+U]\rfloor}\left(A_{i} \wedge L\right)>(1-\eta) t\right] \\
& \leq \mathbb{P}\left[L>t^{1 /(\beta+1)-\varepsilon}\right]+\mathbb{P}\left[\sum_{i=1}^{\lfloor(1-\varepsilon) t / \mathbb{E}[A+U]\rfloor}\left(A_{i} \wedge t^{1 /(\beta+1)-\varepsilon}\right)>(1-\eta) t\right] \\
& =: I_{11}+I_{12} .
\end{aligned}
$$

The condition $\xi>\beta$ implies that, for $0<\varepsilon<(1-\beta / \xi) /(\beta+1)$,

$$
I_{11} \leq O\left(\mathrm{e}^{-t^{(1 /(\beta+1)-\varepsilon) \xi}}\right)=o\left(\mathrm{e}^{-(\log \Phi(t))^{1 /(\beta+1)}}\right) .
$$

Using Chernoff's bound, for $h>0$, we obtain

$$
\begin{aligned}
I_{12} & =\mathbb{P}\left[\exp \left(h\left(\sum_{i=1}^{\lfloor(1-\varepsilon) t / \mathbb{E}[A+U]\rfloor}\left(A_{i} \wedge t^{1 /(\beta+1)-\varepsilon}\right)\right)\right)>\mathrm{e}^{h(1-\eta) t}\right] \\
& \leq \mathrm{e}^{-h(1-\eta) t}\left(\mathbb{E}\left[\mathrm{e}^{h\left(A_{1} \wedge t^{(1 /(\beta+1)-\varepsilon)}\right)}\right]\right)^{\lfloor(1-\varepsilon) t / \mathbb{E}[A+U]\rfloor},
\end{aligned}
$$

which, with $h=\mu t^{-(1 /(\beta+1)-\varepsilon)}, \mu>0,\left(\mathrm{e}^{\mu}-1\right) / \mu<1+\varepsilon / 2$, and using $\mathrm{e}^{x} \leq 1+$ $\left(\mathrm{e}^{b}-1\right) x / b$ for $0 \leq x \leq b$, yields

$$
\begin{aligned}
\mathbb{E}\left[\mathrm{e}^{h\left(A_{1} \wedge t^{(1 /(\beta+1)-\varepsilon)}\right)}\right] & \leq 1+\frac{\mathrm{e}^{\mu}-1}{\mu} h \mathbb{E}\left[\left(A_{1} \wedge t^{(1 /(\beta+1)-\varepsilon)}\right)\right] \\
& \leq 1+\left(1+\frac{\varepsilon}{2}\right) \mu t^{-(1 /(\beta+1)-\varepsilon)} \mathbb{E}\left[A_{1}\right]
\end{aligned}
$$

Then, the preceding inequalities, using $1+x \leq \mathrm{e}^{x}$, imply that

$$
\begin{aligned}
I_{12} & \leq \mathrm{e}^{-\mu(1-\eta) t^{\beta /(\beta+1)+\varepsilon}}\left(1+\left(1+\frac{\varepsilon}{2}\right) \mu t^{-(1 /(\beta+1)-\varepsilon)} \mathbb{E}\left[A_{1}\right]\right)^{(1-\varepsilon) t / \mathbb{E}[A+U]} \\
& \leq \mathrm{e}^{-\mu(1-\eta) t^{\beta /(\beta+1)+\varepsilon}} \mathrm{e}^{(1+\varepsilon / 2) \mu(1-\eta)(1-\varepsilon) t^{\beta /(\beta+1)+\varepsilon}} \\
& =\mathrm{e}^{-\mu(1-\eta)((1+\varepsilon) \varepsilon / 2) t^{\beta /(\beta+1)+\varepsilon}} \\
& =o\left(\mathrm{e}^{-(\log \Phi(t))^{1 /(\beta+1)}}\right)
\end{aligned}
$$

Combining (87) and (88) yields $I_{1}=o\left(\mathrm{e}^{-(\log \Phi(t))^{1 /(\beta+1)}}\right)$ for $\zeta=0$ and $\xi>\beta$. 
Now, in order to prove the $\zeta>0$ case when $\mathbb{P}[A>x]$ is bounded by a Weibull distribution, we need to use the following lemma, which is based on a minor modification of Theorem 3.2(ii) of [5] (or Lemma 2 of [6]) and can be proved by selecting $s=v Q(u) / u, 0<v<1$, in Equation (5.18) of [5], where $Q(u)$ is defined in [5].

Lemma 2. If $\mathbb{P}[A>x] \leq H \mathrm{e}^{-x^{\zeta}}, H>0$ and $1>\zeta>0$, then, for $x^{\theta}<u<\varepsilon x, \varepsilon>0$, $1>\theta>0$, and $n \leq H x$, there exist $C>0$ and $1>\delta>0$ such that

$$
\mathbb{P}\left[\sum_{i=1}^{n} A_{i} \wedge u-n \mathbb{E}[A]>x\right] \leq C \mathrm{e}^{-\delta u^{\zeta-1} x} .
$$

Note that the case $\zeta \geq 1$ is trivial since in this situation $I_{1}$ is exponentially bounded using Chernoff's bound. Therefore, we need to only consider the $0<\zeta<1$ case. Using the union bound and the independence of $\left\{A_{i}\right\}$ and $L$, it is easy to obtain, for $0<\varepsilon<1 /(\beta+1)$,

$$
\begin{aligned}
I_{1}= & \mathbb{P}\left[\sum_{i=1}^{\lfloor(1-\varepsilon) t / \mathbb{E}[A+U]\rfloor}\left(A_{i} \wedge L\right)>(1-\eta) t\right] \\
\leq & \mathbb{P}[L>\varepsilon t]+\mathbb{P}\left[\sum_{i=1}^{\lfloor(1-\varepsilon) t / \mathbb{E}[A+U]\rfloor}\left(A_{i} \wedge t^{1 /(\beta+1)-\varepsilon}\right)>(1-\eta) t\right] \\
& +\int_{t^{1 /(\beta+1)-\varepsilon}}^{\varepsilon t} \mathbb{P}\left[\sum_{i=1}^{\lfloor(1-\varepsilon) t / \mathbb{E}[A+U]\rfloor}\left(A_{i} \wedge u\right)>(1-\eta) t\right] \mathrm{d} \mathbb{P}[L \leq u] \\
= & : I_{11}+I_{12}+I_{13} .
\end{aligned}
$$

From (83) and (88), we obtain

$$
I_{11}+I_{12}=o\left(\mathrm{e}^{-(\log \Phi(t))^{1 /(\beta+1)}}\right) .
$$

Applying Lemma 2 yields, for $t^{1 /(\beta+1)-\varepsilon} \leq u \leq \varepsilon t$,

$$
\begin{aligned}
& \mathbb{P}\left[\sum_{i=1}^{\lfloor(1-\varepsilon) t / \mathbb{E}[A+U]\rfloor}\left(A_{i} \wedge u\right)>(1-\eta) t\right] \\
& \quad \leq \mathbb{P}\left[\sum_{i=1}^{\lfloor(1-\varepsilon) t / \mathbb{E}[A+U]\rfloor}\left(A_{i} \wedge u\right)-\left\lfloor\frac{(1-\varepsilon) t}{\mathbb{E}[A+U]}\right\rfloor \mathbb{E}[A]>\varepsilon(1-\eta) t\right] \\
& \quad \leq C \mathrm{e}^{-\delta \varepsilon(1-\eta) u^{\zeta-1} t},
\end{aligned}
$$

resulting in

$$
\begin{aligned}
I_{13} \leq & \int_{t^{1 /(\beta+1)-\varepsilon}}^{\varepsilon t} C \mathrm{e}^{-\delta \varepsilon(1-\eta) t u^{\zeta-1}} \mathrm{~d} \mathbb{P}[L \leq u] \\
\leq & \left.C \mathrm{e}^{-\delta \varepsilon(1-\eta) t u^{\zeta-1}} \mathbb{P}[L>u]\right|_{\varepsilon t} ^{t^{1 /(\beta+1)-\varepsilon}} \\
& +\int_{t^{1 /(\beta+1)-\varepsilon}}^{\varepsilon t} H \mathrm{e}^{-u^{\xi}} C \mathrm{e}^{-\delta \varepsilon(1-\eta) t u^{\zeta-1}}(1-\zeta) \delta \varepsilon(1-\eta) t u^{\zeta-2} \mathrm{~d} u \\
\leq & \sup _{t^{1 /(\beta+1)-\varepsilon} \leq u \leq \varepsilon t}\left\{C \mathrm{e}^{-u^{\xi}-\delta \varepsilon(1-\eta) t u^{\zeta-1}}\right\}\left(1+O(1) \int_{t^{1 /(\beta+1)-\varepsilon}}^{\varepsilon t} t u^{\zeta-2} \mathrm{~d} u\right) .
\end{aligned}
$$


Since the function $f(u):=C \mathrm{e}^{-u^{\xi}-\delta \varepsilon(1-\eta) t u^{\zeta-1}}$ is absolutely continuous on $\left[t^{1 /(\beta+1)-\varepsilon}, \varepsilon t\right]$, the supremum is obtained either at the two endpoints or when $f^{\prime}(u)=0$. Straightforward calculations show that there is only one point $u^{*}$ where $f^{\prime}\left(u^{*}\right)=0$, and $f\left(u^{*}\right)=$ $O\left(\mathrm{e}^{-h t^{\xi /(\xi+1-\zeta)}}\right)$ for some $h>0$. Furthermore, for some $h>0$, we can verify that $f(u)=$ $O\left(\mathrm{e}^{-h t^{(\xi+\beta) /(\beta+1)+(1-\xi) \varepsilon}}\right)$ when $u=t^{1 /(\beta+1)-\varepsilon}$ and $f(u)=O\left(\mathrm{e}^{-h t^{\xi}}\right)$ when $u=\varepsilon t$. Therefore, by our assumptions $\xi>\beta /(\beta+1)$ and $(1-\zeta) \beta<\xi$, these preceding bounds imply that $I_{13}=o\left(\mathrm{e}^{-(\log \Phi(t))^{1 /(\beta+1)}}\right)$, which, together with (89) and the proof of the $\zeta=0$ case, implies that, for all $\zeta \geq 0$,

$$
I_{1}=o\left(\mathrm{e}^{-(\log \Phi(t))^{1 /(\beta+1)}}\right) .
$$

Thus, combining (82), (83), (84), (86), and (90), and passing $\varepsilon \rightarrow 0$ yields

$$
\varliminf_{t \rightarrow \infty} \frac{\log \mathbb{P}[T>t]^{-1}}{(\log \Phi(t))^{1 /(\beta+1)}} \geq \frac{\beta^{1 /(\beta+1)}+\beta^{-\beta /(\beta+1)}}{(\mathbb{E}[A+U])^{\beta /(\beta+1)}} .
$$

Let us now prove the lower bound. Applying the same argument as used to derive (69) in the proof of the lower bound for Theorem 5, it is easy to obtain, for $\delta>0$,

$$
\mathbb{P}[T>t] \geq \mathbb{P}\left[N \geq \frac{t(1+\delta)}{\mathbb{E}[U+A]}+1\right]-\mathbb{P}\left[\sum_{i=1}^{N-1}\left(U_{i}+A_{i}\right) \leq t, N \geq \frac{t(1+\delta)}{\mathbb{E}[U+A]}+1\right],
$$

where the second probability on the right-hand side is exponentially bounded (see (70)). Therefore, using Theorem 3 and passing $\delta \rightarrow 0$ yields

$$
\varlimsup_{t \rightarrow \infty} \frac{\log \mathbb{P}[T>t]^{-1}}{(\log \Phi(t))^{1 /(\beta+1)}} \leq \frac{\beta^{1 /(\beta+1)}+\beta^{-\beta /(\beta+1)}}{(\mathbb{E}[A+U])^{\beta /(\beta+1)}} .
$$

Combining (91) and (92) completes the proof.

\section{References}

[1] Asmussen, S. et al. (2008). Asymptotic behavior of total times for jobs that must start over if a failure occurs. Math. Operat. Res. 33, 932-944.

[2] Bertsekas, D. P. and Gallager, R. (1992). Data Networks, 2nd edn. Prentice Hall.

[3] Bingham, N. H., Goldie, C. M. And Teugels, J. L. (1987). Regular Variation. Cambridge University Press.

[4] Fiorini, P. M., Sheahan, R. ANd LiPSKy, L. (2005). On unreliable computing systems when heavy-tails appear as a result of the recovery procedure. In ACM SIGMETRICS Performance Evaluation Rev. 33, 15-17.

[5] Jelenković, P. And MomčIlović, P. (2003). Large deviation analysis of subexponential waiting times in a processor-sharing queue. Math. Operat. Res. 28, 587-608.

[6] Jelenković, P. R. And MomčIlović, P. (2004). Large deviations of square root insensitive random sums. Math. Operat. Res. 29, 398-406.

[7] Jelenković, P. R. And TAN, J. (2007). Is ALOHA causing power law delays? In Managing Traffic Performance in Converged Networks (Lecture Notes Comput. 4516), Springer, Berlin, pp. 1149-1160.

[8] Jelenković, P. R. AND TAN, J. (2007). Can retransmissions of superexponential documents cause subexponential delays? In Proc. IEEE INFOCOM '07(New York, May 2007), pp. 892-900.

[9] Jelenković, P. R. and Tan, J. (2007). Characterizing heavy-tailed distributions induced by retransmissions. Preprint. Available at http:// arxiv.org/abs/0709.1138v2.

[10] Kulkarni, V. G., Nicola, V. F. and Trivedi, K. S. (1987). The completion time of a job on a multimode system. Adv. Appl. Prob. 19, 932-954.

[11] Nagaev, S. V. (1979). Large deviations of sums of independent random variables. Ann. Prob. 7, 745-789.

[12] Ross, S. M. (2002). A First Course in Probability, 6th edn. Prentice Hall.

[13] Sheahan, R., Lipsky, L., Fiorini, P. M. and Asmussen, S. (2006). On the completion time distribution for tasks that must restart from the beginning if a failure occurs. In ACM SIGMETRICS Performance Evaluation Rev. 34, 24-26. 